\title{
Locally corrected semi-Lagrangian methods for Stokes flow with moving elastic interfaces
}

\author{
J. Thomas Beale ${ }^{\mathrm{a}, *}$ and John Strain ${ }^{\mathrm{b}}$ \\ ${ }^{a}$ Department of Mathematics, Duke University, Box 90320, Durham, NC \\ 27708-0320 \\ b Department of Mathematics, University of California, 970 Evans Hall \#3840, \\ Berkeley, California 94720-3840
}

\begin{abstract}
We present a new method for computing two-dimensional Stokes flow with moving interfaces that respond elastically to stretching. The interface is moved by semiLagrangian contouring: a distance function is introduced on a tree of cells near the interface, transported by a semi-Lagrangian time step and then used to contour the new interface. The velocity field in a periodic box is calculated as a potential integral resulting from interfacial and body forces, using a technique based on Ewald summation with analytically derived local corrections. The interfacial stretching is found from a surprisingly natural formula. A test problem with an exact solution is constructed and used to verify the speed, accuracy and robustness of the approach.
\end{abstract}

Key words: elastic interfaces, fluid-structure interactions, Stokes equations, Ewald summation, boundary integrals, local corrections

\section{Introduction}

We present a new numerical method for computing time-dependent Stokes flow in two dimensions, with a moving material interface which responds elastically to stretching. The resulting elastic force creates jumps in the fluid velocity gradient and pressure at the interface. We move the interface by semi-Lagrangian

\footnotetext{
* Corresponding author.

Email addresses: beale@math.duke.edu (J. Thomas Beale), strain@math. berkeley.edu (John Strain).

URLs: http://math.duke.edu/faculty/beale (J. Thomas Beale), http://math. berkeley.edu/ strain (John Strain).
} 
contouring [41,40,38,2]: Given the current interface location and velocity, an implicit representation of the interface is constructed by building a signed distance function. The velocity is extended, and the distance function is transported by a second-order semi-Lagrangian formula. The updated interface is found by contouring the zero set of the transported function. The fluid velocity, determined by the interface location and jump conditions, is computed as a potential integral in a periodic box by Ewald summation, which splits the velocity into two parts. The smooth part is calculated by a rapidly-converging Fourier series, while the local part is approximated using asymptotic analysis, resulting in a local correction formula. The velocity can be evaluated accurately at arbitrary points in the fluid region. The stretching of the interface, which determines the elastic force, is evolved by a surprisingly simple formula. We derive an exact solution of the full problem, time-periodic, in which the interface is an ellipse of varying eccentricity. Numerical experiments with this exact solution confirm second-order accuracy. The method is designed so that it can be extended to Navier-Stokes flow and to three-dimensional flow. The immersed boundary method of Peskin [29] has been used extensively to model biological problems in Stokes or Naver-Stokes flow with elastic interfaces, and the immersed interface method is an alternative. These and other methods are discussed below.

We consider fluid flow in a periodic box $Q$, modeled by Stokes flow, that is, viscous, incompressible flow with the material derivative in the usual NavierStokes equations neglected. Stokes flow is an appropriate model at small scales, where viscous forces dominate, and is common in biological problems [29]. The velocity vector $v(x, t)$ and pressure $p(x, t)$ satisfy the equations

$$
-\nu \Delta v+\nabla p=F, \quad \nabla \cdot v=0
$$

with a force

$$
F=f \delta_{\Gamma}+F_{b}
$$

consisting of an interfacial force $f$ on the interface $\Gamma$ and a body force $F_{b}$ defined on the whole box $Q$. The interface $\Gamma$ is a closed curve (or several of them) consisting of elastic material immersed in the fluid and moving with the fluid velocity. The interfacial force is defined distributionally by its action on a test function $w$,

$$
\int_{Q} f(x) \delta_{\Gamma}(x) w(x) \mathrm{d} x=\int_{\Gamma} f(x(s)) w(x(s)) d s
$$

where $s$ is the arclength parameter on $\Gamma$ at the current time. We assume the viscosity is a constant $\nu$ and the fluid density $\rho=1$ inside and outside $\Gamma$; the fluid is the same on both sides.

The interfacial force $f \delta_{\Gamma}$ corresponds to a jump in the normal stress across 
the interface [30],

$$
\left[T_{i j}\right] n_{j}=-f_{i}, \quad i=1,2
$$

with an implied sum over the repeated index $j$. Here $T_{i j}$ is the stress tensor

$$
T_{i j}=-p \delta_{i j}+\nu\left(v_{i, j}+v_{j, i}\right)
$$

square brackets [.] denote the outside value minus the inside, and the normal vector $n$ points outward. Resolving this jump into normal and tangential components gives well-known jump conditions [30,26,27] for $p$ and $\partial v / \partial n$ :

$$
[p]=f \cdot n, \quad \nu\left[\frac{\partial v}{\partial n}\right]=-(f \cdot \tau) \tau
$$

with viscosity $\nu$ constant. We suppose the force $f$ at the interface is determined by surface tension and thus has the form

$$
f=\frac{\partial}{\partial s}(\gamma \tau)
$$

where $\gamma$ is the (variable) coefficient of surface tension and $s$ is the arclength at the current time. (For derivation from classical force balance arguments, see e.g. [24,21,31].) An equivalent form of Eq. (1.7) is

$$
f=(\partial \gamma / \partial s) \tau-\gamma \kappa n
$$

where $\kappa$ is the curvature, defined by $\partial \tau / \partial s=-\kappa n$. We assume that the interfacial tension comes from the elastic response of the interface material, so that $\gamma$ is a function of the form

$$
\gamma=\gamma\left(s_{\alpha}-1\right)
$$

Here $\alpha$ is a material coordinate, equal to arclength in the equilibrium configuration; if the curve is parametrized by $x=X(\alpha, t)$, then

$$
s_{\alpha}=\partial s / \partial \alpha=|\partial X / \partial \alpha|
$$

In our examples we suppose $\gamma$ is linear when the stretching is positive:

$$
\gamma=\gamma_{0}\left(s_{\alpha}-1\right)_{+}=\gamma_{0} \max \left(0, s_{\alpha}-1\right)
$$

Our formulation is the same as that of Peskin [29], except that our $\alpha$ corresponds to Peskin's $s$, and our $f$ differs from his by the factor $s_{\alpha}$. We have included the body force $F_{b}$ to simplify the generation of exact test problems, as was done in [27].

The rest of the paper is organized as follows. Sec. 2 describes the evolution of the stretching factor $s_{\alpha}$. Cottet et al. $[10,11]$ related it to the gradient of 
a signed distance function transported by the physical velocity. Eqs. (2.2) and (2.7) give a generalization, valid for any extension of the physical velocity off the interface, which expresses the evolution of $s_{\alpha}$ in a surprisingly simple way. This generalization incorporates the evolution of $s_{\alpha}$ into the interface motion, without tracking the material coordinate $\alpha$. Sec. 3 summarizes semi-Lagrangian contouring for moving interfaces, and outlines the overall algorithm used in this work. The velocity determined by the Stokes equations is the singular integral of Eq. (3.1), using the fundamental solution in a periodic box. Sec. 4 derives a new Ewald splitting of this periodic fundamental solution, based on Ewald summation of the Green functions for the Laplacian and biharmonic operators. The integral for the Stokes velocity then has two parts, one with a smooth kernel and one with a local kernel. In Sec. 5 approximations are derived for the local contribution from a single layer potential on the curve, or from a body force which has a jump at the curve.

In Sec. 6 we construct a test problem with an exact periodic solution for the elastic interface problem described by Eqs. (1.1), (1.6), (1.7) and (1.11). The interface is an ellipse with time-varying eccentricity. There is a body force which is discontinuous at the interface. We give the derivation of the test problem in the hope that it might provide a useful test in other work. We do not know of an exact solution which has been used for a comparable problem. Sec. 7 presents our numerical results, including tests with the exact solution which unambiguously verify the accuracy of the method. In Sec. 8 possible extensions of this work are discussed.

The most widely used numerical method for biological problems modeled with elastic interfaces in fluids is the immersed boundary method [29]. The fluid velocity is calculated on a grid, and the force on the curve is spread to the grid points by replacing it with a sum of smoothed delta functions with a carefully designed shape. Although the method is generally first-order accurate, a second-order version has been designed for the case where the interface is replaced by a layer of positive thickness [18]. The accuracy of the method is also considered in $[28,30,45,33,17,20]$. In [9] the smoothed delta functions are analytically projected onto the space of divergence-free vector fields, rather than discretizing before projecting, thus removing an important source of error. In the method of $[10,11]$ the location of the curve is found from a signed distance function advected with the fluid; the smoothed delta function is composed with the signed distance function. The general purpose front tracking method introduced in [46] also uses a smoothed delta function.

While the design of the immersed boundary method makes it useful for a variety of realistic problems, in principle better accuracy should be obtained, at least in some cases, by keeping the interface sharp. The primary method available with this property, for the same class of problems, is the immersed interface method of $\mathrm{R}$. LeVeque and Z. Li [25-27]. The velocity is again com- 
puted on a regular grid, using finite differences, but the force at the interface is incorporated through the immersed interface method: jump terms are added to the difference operators when the stencil crosses the boundary. The force on the fluid (1.7)-(1.9) due to the curve must be computed from its configuration. This can be direct if the curve is tracked, although a level set function was used for the curve motion in [27]. Thorough applications of the immersed interface method to rigid and moving boundaries were made in [23,47]. High order accuracy requires a number of correction terms for spatial and temporal derivatives when differences cross the interface. The present work is another approach in which the interface is kept sharp, but the interfacial curve is moved by the semi-Lagrangian contouring method, the velocity is computed from the integral representation, and the force is found in a natural way. We expect these features allow greater flexibility for the treatment of the boundary motion; in particular, the influence of the force on the nearby fluid is less tied to the grid. Recent work for boundary value problems $[7,6]$ has aspects of several of these methods. Higher order accuracy has been achieved for some problems with moving fluid interfaces by other methods, e.g. the coupled level set, volume-of-fluid method of [43].

Boundary integral methods have been used extensively for Stokes flow in free space, in two or three dimensions, often with different viscosities, to model particles, drops, or bubbles $[32,31]$. Usually the force is normal and proportional to curvature, as in the second term in Eq. (1.8). The integral formulation is natural, since the free space kernels are known explicitly, and since for Stokes flow the singular integrals for the velocity are needed only on the moving boundary. In this work we formulate the problem in a periodic box, rather than free space, since we intend for this approach to be used for Navier-Stokes flow, and boundary conditions are necessary in that case. Also for Navier-Stokes the velocity has to be found at points on a regular grid in the fluid region, leading to nearly singular integrals for points near the curve. The present method solves the general problem of computing the Stokes velocity at arbitrary points in periodic geometry. The possible extension from Stokes flow to Navier-Stokes is discussed in Sec. 8. Other methods have been developed for periodic Stokes flow; see [15] and references therein.

In [22] the boundary integral method was used for moving boundaries in Stokes flow with equal arclength parametrization of the interface. With normal force, it was found that the inherent stability requirement for explicit treatment of the boundary motion was $k \leq C h$, where $k$ is the time step and $h$ is the spacing on the boundary. We expect that similar considerations apply with the general force of Eqs. (1.7) and (1.8). However, stiffness may be a more serious problem with Navier-Stokes flow and with more general elastic response [44].

The Ewald splitting of the Stokes velocity kernel is akin to regularization of the kernel of a singular or nearly singular integral with a local correction $[4,3,8]$. In 
fact, for the free space Laplacian, Gaussian regularization as in [4,3] gives the same splitting as the Ewald method [36]. Approximations for the local parts of nearly singular integrals have been derived in $[4,3,16,36]$. A general approach to Ewald splitting and local corrections for systems of elliptic equations has been developed in [42]. Cortez [8] used regularization of the Stokes fundamental solution in a variety of Stokes problems, with corrections from [4] added for forces on curves.

\section{The stretching factor}

We describe the evolution of the stretching factor which occurs in the expression for the elastic boundary force. The moving interface $\Gamma$ is parametrized by a material coordinate $\alpha$, corresponding to arclength in the unstretched position. If $s=s(\alpha, t)$ is the arclength along the curve at the current time, the stretching factor is $s_{\alpha}(\alpha, t)=\left|X_{\alpha}(\alpha, t)\right|$, as in Eq. (1.10).

Suppose a neighborhood of the interface is moved by a velocity field $V$ which agrees with the fluid velocity $v$ on $\Gamma$ but may be different elsewhere. Although the exact evolution of $s_{\alpha}$ depends only on the velocity on the interface, it can be related to the extended velocity $V$ and the signed distance function $\varphi$ near $\Gamma$. Assuming $\Gamma\left(t_{0}\right)$ is sufficiently smooth, each point $x_{0}$ near the interface has the form $x_{0}=X\left(\alpha, t_{0}\right)+\varphi\left(x_{0}, t_{0}\right) n\left(\alpha, t_{0}\right)$ for some $\alpha$, where $n\left(\alpha, t_{0}\right)$ is the unit normal vector at $X\left(\alpha, t_{0}\right)$, according to the Tubular Neighborhood Theorem, and the inverse mapping $(\alpha, \varphi) \mapsto x_{0}$ is differentiable near $\Gamma\left(t_{0}\right)$. (E.g. see [14], Prop. 0.2.) The Jacobian determinant $\operatorname{det}\left(X_{\alpha}, X_{\varphi}\right)$ of the mapping $(\alpha, \varphi) \mapsto x_{0}$ at a point on the curve is $\pm\left|X_{\alpha}\right||n|= \pm\left|X_{\alpha}\right|= \pm s_{\alpha}$, since $X_{\alpha} \perp n$. The sign depends on the conventions for $\alpha, \varphi$ and $n$.

Since the neighborhood evolves in time with velocity $V$, any point $x$ satisfies the ordinary differential equation $d x / d t=V(x, t)$ with initial condition $x\left(t_{0} ; x_{0}\right)=x_{0}$ at time $t_{0}$. Let $J=J\left(x_{0}, t\right)$ be the Jacobian determinant of $\partial x / \partial x_{0}$. We can think of $\alpha, \varphi$ as coordinates for a neighborhood of $\Gamma(t)$ via the composite function $(\alpha, \varphi) \mapsto x_{0} \mapsto x$. The Jacobian of this composite function for $\varphi=0$ is $\pm J s_{\alpha}^{0}$, where $s_{\alpha}^{0}=s_{\alpha}\left(\alpha, t_{0}\right)$. Thus the derivative of the inverse function $x=\left(x_{1}, x_{2}\right) \mapsto(\alpha, \varphi)$ is the inverse matrix of the derivative of $(\alpha, \varphi) \mapsto x$,

$$
\left(\begin{array}{cc}
\partial_{1} \alpha & \partial_{2} \alpha \\
\partial_{1} \varphi & \partial_{2} \varphi
\end{array}\right)=\frac{1}{ \pm J s_{\alpha}^{0}}\left(\begin{array}{rr}
x_{2, \varphi} & -x_{1, \varphi} \\
-x_{2, \alpha} & x_{1, \alpha}
\end{array}\right)
$$

The second row of this formula implies that $|\nabla \varphi|=\left|X_{\alpha}\right| /\left(J s_{\alpha}^{0}\right)$. Solving for $s_{\alpha}=\left|X_{\alpha}\right|$ gives $s_{\alpha}=|\nabla \varphi| J s_{\alpha}^{0}$. For a material point on the interface, the 
marker $\alpha$ is preserved by the flow, so

$$
s_{\alpha}(\alpha, t)=|\nabla \varphi(x, t)| J\left(x_{0}, t\right) s_{\alpha}\left(\alpha, t_{0}\right), \quad x_{0}=X\left(\alpha, t_{0}\right), \quad x=X(\alpha, t) .
$$

Thus the stretching $s_{\alpha}$ at any time $t$ is determined by its initial value, the mapping induced by $V$, and $\nabla \varphi$ (which in turn is determined by the induced mapping). Further simplification depends on the choice of $V$. If $V$ is the physical velocity $v$, with $\nabla \cdot v \equiv 0$, then $J \equiv 1$, and Eq. (2.2) reduces to $s_{\alpha}(\alpha, t)=|\nabla \varphi(x, t)| s_{\alpha}\left(\alpha, t_{0}\right)$ as in Cor. 2.1 of [11], and Lemma 3.1 of [10]. However, we will use a different extension here.

For the present work it is convenient to extend the velocity field $V(x, t)$ so that its normal derivative is zero on the curve at each time $t$ :

$$
(n \cdot \nabla) V(x, t)=0, \quad x \in \Gamma(t) .
$$

Then, the initial condition $|\nabla \varphi| \equiv 1$ at time $t_{0}$ on $\Gamma\left(t_{0}\right)$ guarantees that

$$
|\nabla \varphi(x, t)|=1, \quad x \in \Gamma(t) .
$$

This is essentially Lemma A.1 in [48]; it is readily verified by differentiating $|\nabla \phi|^{2}$ with respect to time and using the transport equation $\varphi_{t}+V \cdot \nabla \varphi=0$. In this case Eq. (2.2) for the stretching reduces to

$$
s_{\alpha}(\alpha, t)=J\left(x_{0}, t\right) s_{\alpha}\left(\alpha, t_{0}\right) .
$$

Moreover, the well-known fact

$$
\frac{d}{d t} J\left(x_{0}, t\right)=\nabla \cdot V(x, t) J\left(x_{0}, t\right) .
$$

and Eq. (2.5) imply the following simple differential stretching law:

$$
\frac{d}{d t} s_{\alpha}(\alpha, t)=\nabla \cdot V(x, t) s_{\alpha}(\alpha, t), \quad x=X(\alpha, t) .
$$

Our algorithm described below updates $s_{\alpha}$ by this law.

For a surface in $\mathbf{R}^{3}$, Eq. (2.2) has a direct analogue with an entirely similar derivation. If the material coordinates are $\alpha=\left(\alpha_{1}, \alpha_{2}\right)$, the stretching factor is $s_{\alpha}=\left|\left(\partial X / \partial \alpha_{1}\right) \times\left(\partial X / \partial \alpha_{2}\right)\right|$. The 3D analogue of Eq. (2.1) has as its third row the equation $\nabla \varphi=\left(X_{\alpha_{1}} \times X_{\alpha_{2}}\right) /\left(J s_{\alpha}^{0}\right)$, leading again to Eq. (2.2).

\section{The numerical method}

Our algorithm evolves the interface $\Gamma$ and the stretching factor $s_{\alpha}$ on the interface. The interface moves with the velocity field $v$, determined from Eq. 
(1.1). The interfacial force in Eqs. (1.7) through (1.9) is determined by $s_{\alpha}$. We extend the interfacial velocity along normal lines, rather than sampling the physical velocity. Thus condition (2.3) holds, and we can use Eq. (2.7) to update $s_{\alpha}$.

The velocity in Stokes flow has an integral representation derived from potential theory, in terms of the (periodic) fundamental solution $S_{i j}$ of the Stokes equations [31]. The velocity $v=\left(v_{1}, v_{2}\right)$ solving Eq. (1.1) in the periodic domain $Q$ is

$$
v_{i}(y)=\int_{\Gamma} \sum_{j=1}^{2} S_{i j}(y-x(s)) f_{j}(s) \mathrm{d} s+\int_{Q} \sum_{j=1}^{2} S_{i j}(y-x) F_{b j}(x) \mathrm{d} x .
$$

This representation is derived in Sec. 4, and an efficient new method for computing the velocity is derived in Secs. 4 and 5 . The velocity on $\Gamma$ suffices to evolve $\Gamma$, but the velocity field can be evaluated at any desired point by integration.

Given an interfacial velocity $v$, the interface is evolved by the four-step semiLagrangian contouring method developed in $[41,40,37,39,38,2]$ :

Implicit representation. A quadtree data structure called the distance tree, consisting of square cells with pointers to nearby elements of $\Gamma$, is built. In the course of this construction, a cell is split if the concentric triple intersects $\Gamma$, the signed distance function $\varphi$ is efficiently computed on the vertices of the tree, and data is stored to permit efficient evaluation of $\varphi$ anywhere in the domain. The tree and $\varphi$ values provide an efficient implicit representation of the interface $\Gamma$. (See Sec. 1.3 of [40], and for more detail in an earlier version, Secs. 3 and 4 of [37].)

Velocity extension. Given the interfacial velocity $v$ on $\Gamma$ and the distance tree, define the extension $V$ on the vertices $x$ (and Steiner points) of the distance tree by

$$
V(x)=v(y),
$$

where $y$ is a nearest point on $\Gamma$ to $x$. Then define $V$ at arbitrary points by interpolation from the tree vertices (and Steiner points). This "numerical Whitney extension" provides an efficient representation of the extended velocity $V$. (See [40], Sec. 2.)

Semi-Lagrangian evolution. Evolve $\varphi$ (and implicitly the interface) along characteristics of the extended velocity $V$, from time $t$ to $t+k$, by the approximate semi-Lagrangian formula

$$
\psi(\widetilde{x})=\varphi(\widetilde{x}-k V(\widetilde{x})) \approx \varphi(\widetilde{x}, t+k) .
$$

The predicted interface $\widetilde{\Gamma}$ at time $t+k$ is then the zero set of $\psi$. (For a second- 
order predictor-corrector version, see [40], Sec. 1.2, or steps (5), (10), and (11) in the algorithm below.)

Contouring. Given the approximate implicit interface representation $\psi$, find its zero set $\widetilde{\Gamma}$ by the following contouring algorithm: Build a new distance tree as if $\psi$ were a signed distance function, by splitting each cell whose edge length exceeds the minimum value of $\psi$ on the cell. Triangulate the resulting tree and find the exact polygonal zero set of the piecewise linear interpolant to the values of $\psi$ at the vertices of the triangulation. Refine the zero set by moving and adding vertices to reduce $|\psi|$ at each interface vertex below a user-specified tolerance. (See [41], Sec. 2.)

Our algorithm for Stokes flow extends the semi-Lagrangian contouring scheme to evolve the function $q \equiv s_{\alpha}$ as well as the interface. (A similar semiLagrangian approach to evolving interface data has been developed in [2], in the context of realistic three-dimensional computer graphics.) One time step of our algorithm evolves $\Gamma$ and $q$ from time $t$ to $t+k$ by the following substeps:

(1) Input at time $t$ : the interface $\Gamma$, the signed distance function $\varphi$ to $\Gamma$, the distance tree $T$, and the stretching factor $q$ on $\Gamma$.

(2) Interfacial force computation: Build a local uniform mesh covering a tubular neighborhood of $\Gamma$. Compute the normal $n$ (or tangent $\tau$ ) and curvature $\kappa$ by high-order ENO differentiation of $\varphi$ on the local mesh. Use Eqs. (1.7) and (1.8) to compute the force $f$ on the interface $\Gamma$.

(3) Interfacial velocity evaluation: From $f$ on $\Gamma$ and body force $F_{b}(t)$, find the interfacial velocity $v$ on $\Gamma$ from Eq. (3.1).

(4) Velocity extension: Extend $v$ to $V$ on the distance tree $T$ built for the interface $\Gamma$.

(5) Contouring: Find $\widetilde{\Gamma}$ as the set of $\widetilde{x}$ with $\varphi(x)=0$, where $x=\widetilde{x}-k V(\widetilde{x})$.

(6) Distancing: Build new distance tree $\widetilde{T}$ and compute $\widetilde{\varphi}$, the signed distance function to $\widetilde{\Gamma}$, on $\widetilde{T}$.

(7) Stretching update: Compute $\nabla \cdot V(x)$ for $x \in \Gamma$ via a local uniform mesh near $\Gamma$. Update $q$ to $\widetilde{q}(\widetilde{x})=(1+k \nabla \cdot V(x)) q(x)$ for $\widetilde{x}$ on $\widetilde{\Gamma}$. (This completes the predictor half-cycle.)

(8) Interfacial force computation: Construct a local uniform mesh near $\widetilde{\Gamma}$. Compute the new normal, tangent, curvature and the force $\widetilde{f}$ determined by $\widetilde{q}$ on the predicted interface $\widetilde{\Gamma}$.

(9) Interfacial velocity evaluation: From $\widetilde{f}$ on $\widetilde{\Gamma}$ and a body force $F_{b}(t+k)$, find the interfacial velocity $\widetilde{v}$ on $\widetilde{\Gamma}$ from Eq. (3.1).

(10) Averaged velocity extension: Extend $\widetilde{v}$ to $\widetilde{V}$ on the distance tree $\widetilde{T}$ built for the curve $\widetilde{\Gamma}$. Construct the second-order averaged velocity on $\widetilde{T}$

$$
\bar{V}(\bar{x})=\frac{1}{2} \tilde{V}(\bar{x})+\frac{1}{2} V(\bar{x}-k V(\bar{x}))
$$

(11) Contouring: Find $\bar{\Gamma}$ as the set of $\bar{x}$ with $\varphi(x)=0$, where $x=\bar{x}-k \bar{V}(\bar{x})$. 
(12) Distancing: Build a new tree $\bar{T}$ and compute $\bar{\varphi}$, the signed distance function to $\bar{\Gamma}$, on $\bar{T}$.

(13) Stretching update: For $x \in \Gamma$ find $\nabla \cdot V(x)$ via a local uniform mesh near $\Gamma$. For $\bar{x} \in \bar{\Gamma}$ find $\nabla \cdot \tilde{V}(\bar{x})$ via a local uniform mesh near $\bar{\Gamma}$ and update $q$ by the second-order formula

$$
\bar{q}(\bar{x})=q(x)+\frac{1}{2} k \nabla \cdot V(x) q(x)+\frac{1}{2} k \nabla \cdot \widetilde{V}(\bar{x}) \bar{q}(\bar{x})
$$

with the last term locally implicit, or equivalently

$$
\bar{q}(\bar{x})=\frac{1+\frac{1}{2} k \nabla \cdot V(x)}{1-\frac{1}{2} k \nabla \cdot \widetilde{V}(\bar{x})} q(x)
$$

Here $x=\bar{x}-k \bar{V}(\bar{x})$ as in step (11). (This ends the complete predictorcorrector cycle.)

\section{Ewald summation for Stokes flow}

Ewald summation is a family of classical techniques for fast summation of periodic Green functions for elliptic operators. Techniques for specific operators such as the Laplacian and Stokes operators have been derived in many classical works $[13,19]$ and applied in many modern computations [1,36,34], while general techniques for elliptic systems have been developed in [42]. We present a new Ewald summation technique for the Stokes equations. It leads to a new local correction scheme remarkably similar to several previous schemes which have been derived from a completely different point of view [4].

\subsection{The steady Stokes equations}

The steady Stokes equations on a $d$-dimensional periodic box $Q=[-\pi, \pi]^{d} \subset$ $R^{d}$ for the velocity $v: Q \rightarrow R^{d}$ and pressure $p: Q \rightarrow R$ are

$$
-\nu \Delta v+\nabla p=F, \quad \nabla \cdot v=0,
$$

where $F$ is a force with mean zero (for consistency). Applying the divergence operator yields $\Delta p=\nabla \cdot F=\partial_{j} F_{j}$, and substituting back in gives a biharmonic equation

$$
\nu \Delta^{2} v_{i}=\partial_{i} \partial_{j} F_{j}-\Delta F_{i}
$$

where $\partial_{i}=\partial / \partial x_{i}$ and repeated indices imply summation. Let

$$
\Delta G=\delta-1 /|Q|, \quad \int_{Q} G \mathrm{~d} x=0,
$$


define the periodic mean-zero Green function for the Laplace equation and

$$
\Delta B=G, \quad \int_{Q} B \mathrm{~d} x=0
$$

define the periodic mean-zero Green function for the biharmonic equation (4.2). Here $|Q|=(2 \pi)^{d}$ is the box volume and $\delta$ is the usual Dirac delta function. Then

$$
\nu v_{i}=\partial_{i} \partial_{j} B \star F_{j}-\Delta B \star F_{i}
$$

where $\star$ denotes the usual convolution of periodic functions:

$$
f \star g(x)=\int_{Q} f(x-y) g(y) \mathrm{d} y
$$

We now seek convenient formulas for $G, B$ and $\partial_{i} \partial_{j} B$.

\subsection{Ewald summation for the Laplacian}

The obvious Fourier series

$$
G(x)=-(2 \pi)^{-d} \sum_{k \neq 0} \frac{1}{|k|^{2}} e^{i k \cdot x}
$$

for $G$ does not converge quickly enough to be useful in computation. Ewald summation expresses $G$ as the sum of two rapidly-converging series, one smooth and one singular but localized. The technique can be derived in a variety of ways which suggest various extensions. The following derivation appears to be new. Let $K_{t}$ be the fundamental solution of the periodic heat equation given in two different forms as

$$
K_{t}(x)=(2 \pi)^{-d} \sum_{k} e^{-t|k|^{2}} e^{i k \cdot x}=(4 \pi t)^{-d / 2} \sum_{m \in Z^{d}} e^{-r_{m}^{2} / 4 t}
$$

where $r_{m}=|x-2 \pi m|$. As $t \rightarrow 0, K_{t}(x) \rightarrow K_{0}(x)=\delta(x)$, a periodic delta function. Note that $K_{t}$ has mean $1 /|Q|$ for all $t \geq 0$. Fix a smoothing parameter $\sigma>0$ and write

$$
G=G \star(\delta-1 /|Q|)=G \star\left(K_{\sigma}-1 /|Q|\right)+G \star\left(\delta-K_{\sigma}\right)
$$

where the fundamental theorem of calculus, the heat equation, and the definition of $G$ give 


$$
\begin{aligned}
G \star\left(\delta-K_{\sigma}\right) & =G \star\left(K_{0}-K_{\sigma}\right) \\
& =-G \star \int_{0}^{\sigma} \partial_{t} K_{t} \mathrm{~d} t \\
& =-G \star \int_{0}^{\sigma} \Delta K_{t} \mathrm{~d} t \\
& =-\int_{0}^{\sigma} K_{t} \mathrm{~d} t+\sigma /|Q| .
\end{aligned}
$$

The integral is sharply peaked in space, so we collect up global terms and local terms separately to write $G$ as the sum $G=G^{F}+G^{L}$ of Fourier and local parts. Here $G^{F}$ is defined as

$$
G^{F}=G \star\left(K_{\sigma}-1 /|Q|\right)+\sigma /|Q|
$$

so that

$$
G^{F}(x)=(2 \pi)^{-d}\left(\sigma-\sum_{k \neq 0} \frac{e^{-\sigma|k|^{2}}}{|k|^{2}} e^{i k \cdot x}\right) .
$$

The $k=0$ term in the Fourier series for $G^{F}$ is nonzero. This does not matter when convolving with a function $F$ which has mean zero, but will make a difference when evaluating pointwise values of the full Green function.

By integration, we find the complementary local part,

$$
\begin{aligned}
G^{L}(x)=-\int_{0}^{\sigma} K_{t}(x) \mathrm{d} t & =-\sum_{m} \int_{0}^{\sigma}(4 \pi t)^{-d / 2} e^{-r_{m}^{2} / 4 t} \mathrm{~d} t \\
& =-\frac{1}{4} \pi^{-d / 2} \sum_{m} r_{m}^{2-d} \Gamma\left(d / 2-1, \frac{r_{m}^{2}}{4 \sigma}\right) .
\end{aligned}
$$

Here $\Gamma(a, z)=\int_{z}^{\infty} e^{-s} s^{a-1} \mathrm{~d} s$ is the usual incomplete gamma function, which is appropriately singular as $z \rightarrow 0$ but decays exponentially as $z \rightarrow \infty$. Thus we can omit the images $2 \pi m, m \neq 0$, whenever $x$ is well inside the box $Q$.

\subsection{Biharmonic Ewald summation by squaring}

The straightforward approach to Ewald summation for the biharmonic equation requires the inverse Fourier transform of $e^{-t|k|^{4}}$, which is difficult to evaluate. Thus we consider two different approaches, squaring and subtraction, both based on the Ewald splitting of the Green function $G$ for the Laplacian. The present work employs subtraction. In the squaring approach, we observe that

$$
\begin{aligned}
B & =G \star G=\left(G^{F}+G^{L}\right) \star\left(G^{F}+G^{L}\right) \\
& =G^{F} \star\left(G^{F}+2 G^{L}\right)+G^{L} \star G^{L} \equiv B^{F}+B^{L}
\end{aligned}
$$


where (since convolution multiplies Fourier coefficients)

$$
B^{F}(x)=(2 \pi)^{-d}\left(-\sigma^{2}+\sum_{k \neq 0} \frac{e^{-\sigma|k|^{2}}\left(2-e^{-\sigma|k|^{2}}\right)}{|k|^{4}} e^{i k \cdot x}\right)
$$

and

$$
B^{L}(x)=\int_{0}^{\sigma} \int_{0}^{\sigma}\left(K_{t} \star K_{s}\right)(x) \mathrm{d} s \mathrm{~d} t=\int_{0}^{\sigma} \int_{0}^{\sigma} K_{t+s}(x) \mathrm{d} s \mathrm{~d} t
$$

since the heat kernel defines a semigroup. Consider $G^{L}(x ; \sigma)$ as a function of the smoothing parameter $\sigma$, (as well as $x$ ) and let $H(x ; t)=\int_{0}^{t} G^{L}(x ; \sigma) d \sigma$ be an antiderivative; then

$$
B^{L}(x)=\int_{0}^{\sigma} \partial_{t} H(x ; t+\sigma)-\partial_{t} H(x ; t) \mathrm{d} t=H(2 \sigma)-2 H(\sigma)
$$

where a tedious calculation gives

$$
H(t)=\frac{1}{16 \pi^{d / 2}} \sum_{m} r_{m}^{4-d}\left(\frac{4 t}{r_{m}^{2}} \Gamma\left(d / 2-1, \frac{r_{m}^{2}}{4 t}\right)+\Gamma\left(d / 2-2, \frac{r_{m}^{2}}{4 t}\right)\right)
$$

The occurrence of four separate incomplete gamma functions in expression (4.18) is computationally inconvenient and complicates local correction formulas such as Eq. (5.34) below, so we also employ another approach which leads to simpler formulas.

\subsection{Biharmonic Ewald summation by subtraction}

We also derive a more convenient approach in which the local part of the biharmonic Ewald sum is defined by modifying the free-space Green function in a way that preserves the local singularity. We take the two-dimensional case $d=2$ for simplicity. The free-space Green function for the Laplace equation is

$$
G_{\infty}(x)=\frac{1}{2 \pi} \log |x|,
$$

and the corresponding free-space Green function for the biharmonic is

$$
B_{\infty}(x)=\frac{1}{8 \pi}|x|^{2}(\log |x|-1) .
$$

The derivation of harmonic Ewald summation and the singularity

$$
\Gamma(0, z)=\int_{z}^{\infty} e^{-s} \frac{d s}{s} \sim-\log z \quad \text { as } z \rightarrow 0
$$


suggests that we define

$$
B^{L}(x)=-\frac{1}{16 \pi} \sum_{m \in Z^{2}} r_{m}^{2} \Gamma\left(0, \frac{r_{m}^{2}}{4 \sigma}\right)
$$

to provide a periodic rapidly-decaying function which matches the local singularity of $B$. We then use the Poisson summation formula to evaluate the smooth remainder $B^{F}=B-B^{L}$ as a Fourier series. Neither $B^{F}$ nor $B^{L}$ have

mean zero, but their sum $B$ does. Reversing the computation of $G^{L}(x)$ shows that

$$
\begin{aligned}
B^{L}(x) & =-\frac{1}{4} \int_{0}^{\sigma} \sum_{m} r_{m}^{2}(4 \pi t)^{-1} e^{-r_{m}^{2} / 4 t} \mathrm{~d} t \\
& =-\frac{1}{4 \pi} \int_{0}^{\sigma} t \frac{d}{d t} \sum_{m} e^{-r_{m}^{2} / 4 t} \mathrm{~d} t \\
& =-(2 \pi)^{-2} \sum_{k} \int_{0}^{\sigma} t \frac{d}{d t}\left(t e^{-t|k|^{2}}\right) \mathrm{d} t e^{i k \cdot x}
\end{aligned}
$$

where we have integrated by parts and used the equality (4.8), a special case of the Poisson summation formula [12]. Then, since $B^{F}=B-B^{L}$ and

$$
B(x)=(2 \pi)^{-2} \sum_{k \neq 0} \frac{1}{|k|^{4}} e^{i k \cdot x}
$$

we obtain the following rapidly-converging Fourier series representation:

$$
B^{F}(x)=(2 \pi)^{-2}\left(\frac{\sigma^{2}}{2}+\sum_{k \neq 0} \frac{1}{|k|^{4}}\left(1+\sigma|k|^{2}+\sigma^{2}|k|^{4}\right) e^{-\sigma|k|^{2}} e^{i k \cdot x}\right) .
$$

Its second partial derivatives are given by

$$
\partial_{i} \partial_{j} B^{F}(x)=-(2 \pi)^{-2} \sum_{k \neq 0} \frac{k_{i} k_{j}}{|k|^{4}}\left(1+\sigma|k|^{2}+\sigma^{2}|k|^{4}\right) e^{-\sigma|k|^{2}} e^{i k \cdot x} .
$$

\subsection{Application to the Stokes velocity}

The decomposition $B=B^{F}+B^{L}$ above leads to a corresponding decomposition of the operator producing the Stokes velocity into a smooth part and a local part. We write Eq. (4.5) in the form

$$
\nu v_{i}=\Sigma_{j} S_{i j} \star F_{j}
$$

with

$$
S_{i j}=\partial_{i} \partial_{j} B-\delta_{i j} \Delta B
$$


It is natural to write $S_{i j}$ as the sum

$$
S_{i j}=S_{i j}^{F}+S_{i j}^{L}
$$

with

$$
S_{i j}^{F}=\partial_{i} \partial_{j} B^{F}-\delta_{i j} \Delta B^{F}, \quad S_{i j}^{L}=\partial_{i} \partial_{j} B^{L}-\delta_{i j} \Delta B^{L} .
$$

The smooth part $S^{F}$ has the Fourier representation

$$
\begin{aligned}
S_{i j}^{F}(x) & =(2 \pi)^{-2} \sum_{k \neq 0} s_{i j}(k) e^{i k \cdot x} \\
s_{i j}(k) & =\frac{\delta_{i j}|k|^{2}-k_{i} k_{j}}{|k|^{4}}\left(1+\sigma|k|^{2}+\sigma^{2}|k|^{4}\right) e^{-\sigma|k|^{2}} .
\end{aligned}
$$

The local part will be written explicitly in the next section.

\section{Computation of the Stokes velocity}

In the last section the velocity $v$, determined by the Stokes equations (4.1) with given force $F$, was written as the convolution

$$
\nu v_{i}=\sum_{j} S_{i j} \star F_{j} .
$$

The velocity kernel $S$ given by Eq. (4.28) was decomposed into a smooth part and a local part, $S=S^{F}+S^{L}$. The two parts will be treated separately. For convenience we write the velocity as $v=v^{F}+v^{L}$.

\subsection{The Fourier part}

The velocity term $v^{F}$ contributed by the smooth part of the kernel is computed as a Fourier series. The method is similar to that of [36]. Writing $F$ in a Fourier series,

$$
F(x)=\sum_{k} \hat{F}(k) e^{i k x}, \quad \hat{F}(k)=(2 \pi)^{-2} \int_{Q} F(x) e^{-i k x} \mathrm{~d} x
$$

and using the Fourier representation (4.30) of $S^{F}$, we have

$$
\nu v_{i}^{F}(x)=\sum_{j} \sum_{k} s_{i j}(k) \hat{F}_{j}(k) e^{i k x} .
$$

Because of the exponential decay of $s_{i j}(k)$ in Eq. (4.31), we can truncate the series. We choose a truncation index $p$ and sum over $k=\left(k_{1}, k_{2}\right)$ with 
$\left|k_{1}\right|,\left|k_{2}\right| \leq p$. The error $E_{F}$ due to this truncation is at most

$$
\left|E_{F}\right| \leq \sqrt{2 \pi} \frac{e^{-p^{2} \sigma}}{p^{3} \sigma^{3 / 2}}
$$

(See [36], p. 253.) The error bound (5.4) depends only on $p^{2} \sigma$ and decays rapidly as $p^{2} \sigma \rightarrow \infty$. For example, $p^{2} \sigma=20$ gives an error less than $10^{-10}$.

If the force $F$ is on the curve, in the form $F=f \delta_{\Gamma}$, the Fourier coefficient of $F$ is an integral over the curve,

$$
\hat{F}(k)=(2 \pi)^{-2} \int_{\Gamma} f(x) e^{-i k x} \mathrm{~d} s(x) .
$$

We compute these coefficients by a quadrature rule over the line segments of which $\Gamma$ is constructed. (Compare [36], p. 255.) If $F$ is a body force, we use a trapezoidal rule for the integral (5.5). If $F$ has a jump discontinuity at $\Gamma$, we approximate $\Gamma$ by a line segment in each grid square it cuts and choose some value of $F$ on the correct side for each part of the square. The resulting Fourier coefficients are second-order accurate in the grid spacing. The complexity of the computation is cubic in the grid spacing, since each interface element affects every Fourier coefficient; thus optimal complexity requires geometric nonuniform FFT techniques [35].

\subsection{The local part}

We derive approximations to the contribution $v^{L}$ valid for small $\sigma$. This is possible because the kernel $S^{L}$ decays like a Gaussian away from the singularity. It is convenient to replace the Ewald parameter $\sigma$ with a new parameter $\delta$, defined by

$$
\delta^{2}=4 \sigma
$$

It is the radius of smoothing in $G^{F}$ and $B^{F}$ and the length scale for the decay in $S^{L}$. We obtain an expansion in powers of $\delta$. Similar expansions have been used in [16], [36], [4], [3]. The analytical technique here is close to that in [4].

We ignore the periodic reflections in the formulas (4.13) and (4.22) for $G^{L}$ and $B^{L}$, presuming that the sources are well inside the periodic boundary. Thus, with $r=|x|$,

$$
G^{L}(r) \approx-\frac{1}{4 \pi} \Gamma\left(0, r^{2} / \delta^{2}\right), \quad B^{L}(r) \approx-\frac{r^{2}}{16 \pi} \Gamma\left(0, r^{2} / \delta^{2}\right) .
$$

Corresponding to $B^{L}$, the local part of the Stokes velocity is $v_{i}^{L}=\Sigma_{j} S_{i j}^{L} \star F_{j}$ with

$$
S_{i j}^{L}=\partial_{i} \partial_{j} B^{L}-\delta_{i j} \Delta B^{L}
$$


To obtain detailed expressions we start with derivatives of $G^{L}, B^{L}$ :

$$
\begin{gathered}
\partial_{r} G^{L}=\frac{1}{2 \pi r} e^{-r^{2} / \delta^{2}} \\
\partial_{j} B^{L}=\frac{1}{4}\left(2 x_{j} G^{L}+\frac{x_{j}}{2 \pi} e^{-r^{2} / \delta^{2}}\right)
\end{gathered}
$$

and with $\rho=r / \delta$,

$$
\partial_{i} \partial_{j} B^{L}=\delta_{i j}\left(\frac{1}{2} G^{L}+\frac{e^{-\rho^{2}}}{8 \pi}\right)+\frac{x_{i} x_{j}}{4 \pi r^{2}}\left(1-\rho^{2}\right) e^{-\rho^{2}}
$$

and in particular

$$
\Delta B^{L}=\sum_{j} \partial_{j}^{2} B^{L}=G^{L}+\frac{1}{2 \pi}\left(1-\frac{\rho^{2}}{2}\right) e^{-\rho^{2}} .
$$

Now substituting Eqs. (5.11) and (5.12) in Eq. (4.28) we can write $S^{L}$ explicitly as

$$
S_{i j}^{L}=-\frac{\delta_{i j}}{2} G^{L}+\delta_{i j} e^{-\rho^{2}}\left(-\frac{3}{8 \pi}+\frac{\rho^{2}}{4 \pi}\right)+\frac{x_{i} x_{j}}{4 \pi r^{2}}\left(1-\rho^{2}\right) e^{-\rho^{2}}
$$

To proceed further we consider separately the two types of force.

\subsection{The local integral over a curve}

Consider the local part $v^{L}$ of the velocity induced by a force $F=f \delta_{\Gamma}$ on a curve $\Gamma$. At an arbitrary point $y$

$$
\nu v_{i}^{L}(y)=\int_{\Gamma} \sum_{j} S_{i j}^{L}(y-x(s)) f_{j}(s) d s
$$

where $s$ is the arclength parameter on the curve. We will derive an approximation valid to $O\left(\delta^{3}\right)$. Since $S^{L}$ is localized we are only concerned with $y$ near the curve $\Gamma$ and the part of the integral with $x(s)$ near $y$. For such $y$ we can write $y=x\left(s_{0}\right)+b n\left(s_{0}\right)$ where $x\left(s_{0}\right)$ is a point on $\Gamma$ and $n\left(s_{0}\right)$ is the unit normal at that point. For simplicity we assume $s_{0}=0$. We write $\tau=x^{\prime}(0)$ for the unit tangent, and $n=n(0)$ for the normal, denoting the $s$-derivative with 1. We have $x^{\prime \prime}(0)=-\kappa n$, where $\kappa$ is the curvature at $x(0)$. (See Fig. 1.)

We use Taylor expansions in $s$ and $b$ to identify the largest terms in Eq. (5.14). We begin with

$$
x(s)-y=\tau s-\left(\frac{1}{2} \kappa s^{2}+b\right) n+O\left(s^{3}\right) .
$$

Let $r^{2}=|x(s)-y|^{2}$ and $R^{2}=s^{2}+b^{2}$. Then

$$
r^{2}=s^{2}+b^{2}+\kappa b s^{2}+O\left(R^{4}\right)
$$




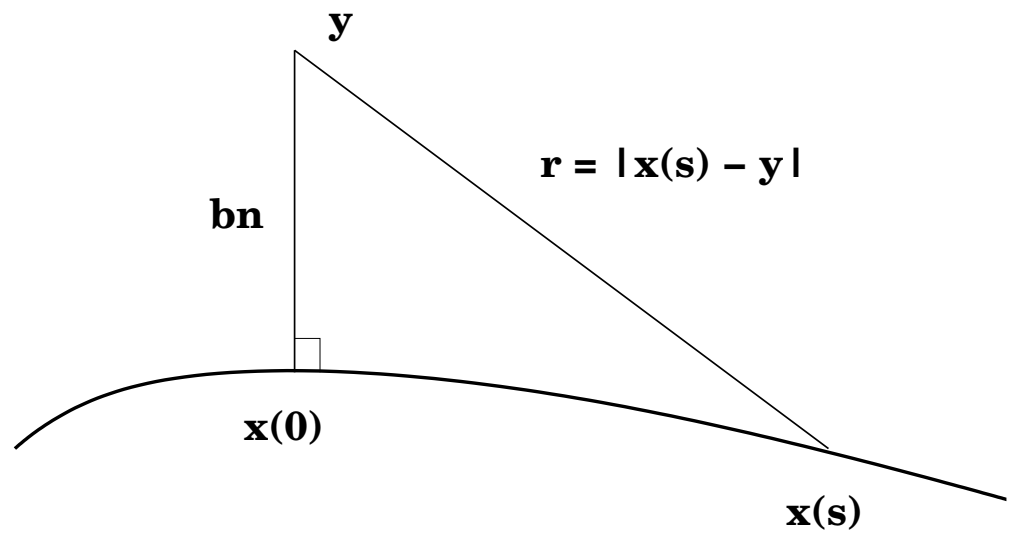

Fig. 1. Integral over the curve evaluated at $y$

We now introduce a new variable $\xi=\xi(s, b)$ defined by $r^{2}=\xi^{2}+b^{2}$ in order to simplify the dependence of the integrand on $r$. Solving for $\xi$ in Eq. (5.16) gives $\xi^{2}=(1+\kappa b) s^{2}+O\left(R^{4}\right)$ or

$$
\xi=\left(1+\frac{1}{2} \kappa b\right) s+O\left(R^{3}\right) .
$$

We can now solve for $s=s(\xi, b)$, obtaining

$$
s=\left(1-\frac{1}{2} \kappa b\right) \xi+O\left(r^{3}\right)
$$

and

$$
\frac{d s}{d \xi}=1-\frac{1}{2} \kappa b+O\left(r^{2}\right) .
$$

Now substituting for $s$ in terms of $\xi$ in Eq. (5.15) gives

$$
x(s)-y=\left(1-\frac{1}{2} \kappa b\right) \xi \tau-\left(\frac{1}{2} \kappa \xi^{2}+b\right) n+O\left(r^{3}\right)
$$

and similarly for $f$

$$
f=f(0)+f^{\prime}(0) s+O\left(s^{2}\right)=f(0)+f^{\prime}(0) \xi+O\left(r^{2}\right) .
$$

In evaluating various parts of the integral (5.14) we use the substitution $\xi=\delta \zeta$ and $b=\delta \beta$, noting that $\rho=r / \delta=\sqrt{\zeta^{2}+\beta^{2}}$. Four integrals related to the Gaussian will be needed. If

$$
I_{p}=\int_{-\infty}^{\infty} \frac{e^{-\left(\zeta^{2}+\beta^{2}\right)}}{\zeta^{2}+\beta^{2}} \zeta^{p} d \zeta, \quad J_{p}=\int_{-\infty}^{\infty} e^{-\left(\zeta^{2}+\beta^{2}\right)} \zeta^{p} d \zeta
$$

then

$$
\begin{gathered}
I_{0}=(\pi /|\beta|) \operatorname{erfc}|\beta|, \quad I_{2}=\sqrt{\pi} e^{-\beta^{2}}-\pi|\beta| \operatorname{erfc}|\beta|, \\
J_{0}=\sqrt{\pi} e^{-\beta^{2}}, \quad J_{2}=(\sqrt{\pi} / 2) e^{-\beta^{2}} .
\end{gathered}
$$


We begin with the integral of $G^{L} f_{i}$, corresponding to the part of Eq. (5.14) coming from the first term of Eq. (5.13)

$$
\begin{array}{r}
\int G^{L}(r / \delta) f_{i} \frac{d s}{d \xi} \mathrm{d} \xi=\int G^{L}(r / \delta)\left[\left(f_{0 i}+f_{0 i}^{\prime} \xi\right)\left(1-\frac{1}{2} \kappa b\right)++O\left(r^{2}\right)\right] \mathrm{d} \xi \\
=\left(1-\frac{1}{2} \kappa b\right) f_{0 i} \int G^{L}(r / \delta) \mathrm{d} \xi+\text { odd term + remainder }
\end{array}
$$

We evaluate the last integral as follows:

$$
\begin{aligned}
\int G^{L}(r / \delta) d \xi & =\delta \int G^{L}(\rho) d \zeta \\
& =-\delta \int \frac{\partial G^{L}}{\partial \zeta} \zeta d \zeta \\
& =-\delta \int \frac{\partial G^{L}}{\partial \rho} \frac{\zeta}{\rho} \cdot \zeta d \zeta \\
& =-\delta \int \frac{1}{2 \pi \rho} e^{-\rho^{2}} \frac{\zeta^{2}}{\rho} d \zeta \\
& =-\frac{\delta}{2 \pi} I_{2} .
\end{aligned}
$$

The term in Eq. (5.25) with $f_{0 i}^{\prime} \xi$ is odd in $\xi$ and integrates to 0 . The $O\left(r^{2}\right)$ remainder in Eq. (5.25) is a sum of terms, each a monomial of degree 2 in $\xi, b$ times a bounded function, and the integral is $O\left(\delta \cdot \delta^{2}\right)=O\left(\delta^{3}\right)$. Thus

$$
A_{1 i} \equiv \int G^{L}(r / \delta) f_{i} d s=-\frac{\delta}{2 \pi}\left(1-\frac{1}{2} \kappa b\right) I_{2} f_{0 i}+O\left(\delta^{3}\right)
$$

Looking at the next terms in Eq. (5.13) we see in a similar but simpler way that

$$
\begin{gathered}
A_{2 i} \equiv \int e^{-\rho^{2}} f_{i} d \xi=\delta\left(1-\frac{1}{2} \kappa b\right) J_{0} f_{0 i}+O\left(\delta^{3}\right) \\
A_{3 i} \equiv \int e^{-\rho^{2}} \rho^{2} f_{i} d \xi=\delta\left(1-\frac{1}{2} \kappa b\right)\left(J_{2}+\beta^{2} J_{0}\right) f_{0 i}+O\left(\delta^{3}\right) .
\end{gathered}
$$

To handle the remaining part of $S^{L}$, we note that

$$
\sum_{j}\left(x_{j}-y_{j}\right) f_{j}=\left(1-\frac{1}{2} \kappa b\right)\left(f_{\tau} \xi+f_{\tau}^{\prime} \xi^{2}\right)-\left(\frac{1}{2} \kappa \xi^{2}+b\right)\left(f_{n}+f_{n}^{\prime} \xi\right)+O\left(r^{3}\right)
$$

where $f_{\tau}=\sum_{j} f_{0 j} \tau_{j}, f_{n}=\sum_{j} f_{0 j} n_{j}$ and similarly ${f^{\prime}}^{\prime}, f^{\prime}{ }_{n}$ are tangential and normal components of $f_{0}^{\prime}$. Then

$$
\begin{aligned}
\sum_{j}\left(x_{i}-y_{i}\right)\left(x_{j}-y_{j}\right) f_{j} \frac{d s}{d \xi} & =\left(1-\frac{3}{2} \kappa b\right) \xi^{2} f_{\tau} \tau_{i}+\left(b^{2}-\frac{1}{2} \kappa b^{3}+b \kappa \xi^{2}\right) f_{n} n_{i} \\
& -b \xi^{2}\left(f^{\prime}{ }_{n} \tau_{i}+{f^{\prime}}^{\prime} n_{i}\right)+\text { odd terms }+O\left(r^{4}\right)(5 .
\end{aligned}
$$


and proceeding as with the earlier terms we find, with $M_{p}=I_{p}-J_{p}$,

$$
\begin{aligned}
A_{4 i} & \equiv \int \sum_{j} \frac{\left(x_{i}-y_{i}\right)\left(x_{j}-y_{j}\right)}{r^{2}}\left(1-\rho^{2}\right) e^{-\rho^{2}} f_{j} d \xi \\
& =\delta\left(1-\frac{3}{2} \kappa b\right) M_{2} f_{\tau} \tau_{i}+\delta \beta^{2}\left(1-\frac{1}{2} \kappa b\right) M_{0} f_{n} n_{i}+\delta^{2} \beta \kappa M_{2} f_{n} n_{i} \\
& -\delta^{2} \beta M_{2}\left(f^{\prime}{ }_{n} \tau_{i}+f^{\prime}{ }_{\tau} n_{i}\right)+O\left(\delta^{3}\right) .
\end{aligned}
$$

We can now substitute Eqs. (5.27-5.29) and (5.32) into Eqs. (5.13) and (5.14) to get an expression for the local part of the velocity,

$$
\nu v_{i}^{L}=-\frac{1}{2} A_{1 i}-\frac{3}{8 \pi} A_{2 i}+\frac{1}{4 \pi} A_{3 i}+\frac{1}{4 \pi} A_{4 i}+O\left(\delta^{3}\right) .
$$

It is convenient to combine the first three terms and rewrite this as

$$
4 \pi \nu v_{i}^{L}=\delta\left(1-\frac{1}{2} \kappa b\right)\left(I_{2}-\left(1-\beta^{2}\right) J_{0}\right) f_{0 i}+A_{4 i}+O\left(\delta^{3}\right) .
$$

In the special case when $b=0$, i.e. $y$ is on the curve, this expression simplifies to

$$
4 \pi \nu v_{i}^{L}=\frac{1}{2} \delta \sqrt{\pi} f_{\tau} \tau_{i}+O\left(\delta^{3}\right)
$$

\subsection{The local integral of a body force}

Next we approximate the local velocity resulting from a body force $F$ which may be discontinuous across a curve $\Gamma$. We first suppose that $F$ is nonzero only inside the domain $D$ bounded by $\Gamma$. In place of Eq. (5.14) we have the integral

$$
\nu v_{i}^{L}(y)=\int_{D} \sum_{j} S_{i j}^{L}(y-x) F_{j}(x) \mathrm{d} x .
$$

If $y$ is away from $\Gamma$, it can be seen that this local velocity is $O\left(\delta^{4}\right)$ because of the form (5.8) expressing $S^{L}$ in terms of second derivatives of $B^{L}$, the fact that $B^{L}$ in Eq. (5.7) is $\delta^{2}$ times a function of $\rho=r / \delta$, and the rapid decay of $B^{L}$. Thus we consider a point $y$ near the curve. For simplicity we assume $y$ is along a vertical normal line to the curve at distance $b$, and we set $y=(0,0)$ without loss of generality. Thus we suppose the curve has the form

$$
x_{2}=Y\left(x_{1}\right)=-b+\frac{1}{2} Y_{0}^{\prime \prime} x_{1}^{2}+O\left(x_{1}^{3}\right)
$$

so that the curve passes through $(0,-b)$ with normal $(0,1)$. (See Fig. 2 .)

The integrand is significant only for $x$ near 0 . Assuming $b$ is small, we will introduce new coordinates $\xi=\left(\xi_{1}, \xi_{2}\right)$ so that the exterior $x_{2}>Y\left(x_{1}\right)$ corresponds to $\xi_{2}>-b$ and the radial distance is preserved, that is,

$$
\xi_{1}^{2}+\xi_{2}^{2}=x_{1}^{2}+x_{2}^{2} \equiv r^{2}
$$




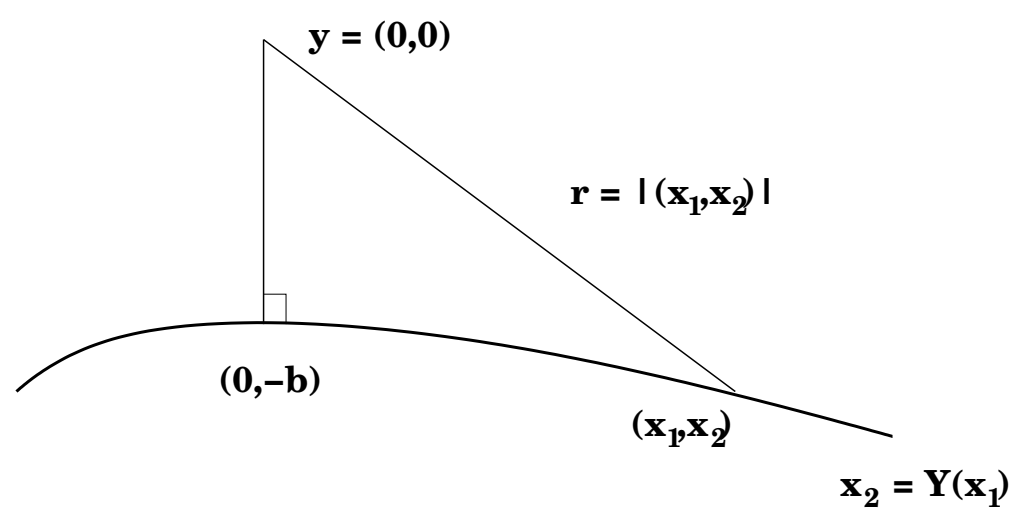

Fig. 2. Integral over the region inside the curve

To do this, given $x$, we first define $\xi_{2}$ as

$$
\xi_{2}=x_{2}-Y\left(x_{1}\right)-b=x_{2}-\frac{1}{2} Y_{0}^{\prime \prime} x_{1}^{2}+O\left(x_{1}^{3}\right)
$$

so that Eq. (5.38) reduces to $\xi_{1}^{2}=x_{1}^{2}+2 \xi_{2}(Y+b)+(Y+b)^{2}$. Since $(Y+b) / x_{1}^{2}$ is smooth, this equation defines $\xi_{1}$, with the convention that $\xi_{1}$ and $x_{1}$ have the same sign. We have $\xi_{1}^{2}=x_{1}^{2}\left(Y_{0}^{\prime \prime} \xi_{2}+O(x)\right)$ or

$$
\xi_{1}=\left(1+\frac{1}{2} Y_{0}^{\prime \prime} \xi_{2}\right) x_{1}+O\left(x^{2}\right) .
$$

From the approximations (5.39) and (5.40) it follows that

$$
x=\xi+O\left(r^{2}\right), \quad J \equiv \operatorname{det}\left(\frac{\partial x}{\partial \xi}\right)=1+O(r) .
$$

We now convert the integral (5.36) to the new variables,

$$
\nu v_{i}^{L}=\int_{-\infty}^{-b} \int_{-\infty}^{\infty} \sum_{j} S_{i j}^{L}(x) F_{j}(x) J d \xi_{1} d \xi_{2}
$$

and note that the dependence on $r / \delta$ is unaffected by the conversion. We will evaluate to $O\left(\delta^{3}\right)$ with crude approximations, but it will be clear that further terms are of the order neglected. Thus we replace $F_{j}(x)$ with $F_{0 j}=F_{j}(0,0)$ and $J$ with 1 . We ignore the distinction between $x$ and $\xi$ to get

$$
\nu v_{i}^{L} \approx \int_{-\infty}^{-b} \int_{-\infty}^{\infty} \sum_{j} S_{i j}^{L}(x) F_{0 j} \mathrm{~d} x .
$$

For $i=2$ we can use Eq. (5.8) to write the integrand as $\partial_{21} B^{L} F_{01}-\partial_{11} B^{L} F_{02}$; since this is an $x_{1}$-derivative, the integral is zero. For $i=1$, the integrand is $\partial_{12} B^{L} F_{02}-\partial_{22} B^{L} F_{01}$; the first term again integrates to zero, and for the same reason we can replace the second term by $-\left(\Delta B^{L}\right) F_{01}$. We now have

$$
\nu v_{1}^{L} \approx-F_{01} \int_{-\infty}^{-b} \int_{-\infty}^{\infty} \Delta B^{L} \mathrm{~d} x
$$


One term in $\Delta B^{L}$ is $G^{L}$. We rewrite the $x_{1}$-integral of this term as

$$
\int_{-\infty}^{\infty} G^{L}\left(x_{1}, x_{2}\right) \mathrm{d} x_{1}=-\int_{-\infty}^{\infty} x_{1} \frac{d G^{L}}{d x_{1}} \mathrm{~d} x_{1}=-\int_{-\infty}^{\infty} \frac{x_{1}^{2}}{2 \pi r^{2}} e^{-r^{2} / \delta^{2}} \mathrm{~d} x_{1} .
$$

Substituting from Eq. (5.12) and combining terms we have

$$
\nu v_{1}^{L} \approx \frac{F_{01}}{2 \pi} \int_{-\infty}^{-b} \int_{-\infty}^{\infty}\left(\frac{-x_{2}^{2}}{x_{1}^{2}+x_{2}^{2}}+\frac{1}{2}\left(x_{1}^{2}+x_{2}^{2}\right)\right) e^{-r^{2} / \delta^{2}} \mathrm{~d} x_{1} \mathrm{~d} x_{2} .
$$

We now rescale the variables by $\left(x_{1}, x_{2}\right) \mapsto\left(\delta x_{1}, \delta x_{2}\right)$ and set $\beta=b / \delta$, so that

$$
\nu v_{1}^{L} \approx \frac{\delta^{2} F_{01}}{2 \pi} \int_{-\infty}^{-\beta} \int_{-\infty}^{\infty}\left(\frac{-x_{2}^{2}}{x_{1}^{2}+x_{2}^{2}}+\frac{1}{2}\left(x_{1}^{2}+x_{2}^{2}\right)\right) e^{-r^{2}} \mathrm{~d} x_{1} \mathrm{~d} x_{2} .
$$

We note that the integrand is even in $x_{2}$ and the integral from 0 to $\infty$ is zero. Thus for $\beta>0$, the integral from $-\infty$ to $-\beta$ is minus that from 0 to $\beta$, and for $\beta<0$, the integral is equal to that from 0 to $|\beta|$. Evaluating the integral we find

$$
\nu v_{1}^{L} \approx-\frac{\delta^{2} F_{01}}{8 \pi} \beta\left(\sqrt{\pi} e^{-\beta^{2}}-2 \pi|\beta| \operatorname{erfc}|\beta|\right) .
$$

The term just found was $O\left(\delta^{2}\right)$ because of the rescaling of the area form. If we considered further terms we would have additional factors of $\xi$, which after rescaling would contribute terms to the integral of order $\delta^{p} \cdot \delta^{2}$ with $p \geq 1$. Returning to general coordinates, we summarize the conclusion for the local velocity (5.36): For a point $y=x+b n$ near the curve, where $n$ is the normal at the nearest point $x$ on the curve to $y$,

$$
\nu v^{L}=-\frac{\delta^{2}}{8 \pi} \beta\left(\sqrt{\pi} e^{-\beta^{2}}-2 \pi|\beta| \operatorname{erfc}|\beta|\right) F_{\tau} \tau+O\left(\delta^{3}\right)
$$

where $\beta=b / \delta, \tau$ is the tangent at $x$, and $F_{\tau}$ is the tangential component of $F$ at $x$.

Finally, we consider the case of an integral over the full periodic domain but with a discontinuity in $F$ at the curve $\Gamma$. In the analysis above, if our integral were outside $D$ rather than inside, the $y$-integral would have been from $-b$ to $\infty$ rather than $-\infty$ to $-b$. The result would be the same except for a change of sign. Thus, for an integral with discontinuity across $\Gamma$, the result would be the difference of two terms as in Eq. (5.49)

$$
\nu v^{L}=\frac{\delta^{2}}{8 \pi} \beta\left(\sqrt{\pi} e^{-\beta^{2}}-2 \pi|\beta| \operatorname{erfc}|\beta|\right)\left[F_{\tau}\right] \tau+O\left(\delta^{3}\right)
$$

where $\left[F_{\tau}\right]$ means the outside value minus the inside of $F_{\tau}$. 


\section{An exact solution with elastic force}

We construct an exact solution in a $2 \pi$-periodic box $Q$, for which the elastic interface is a moving ellipse

$$
x=a(t) \cos \theta, \quad y=b(t) \sin \theta
$$

parametrized by $\theta$, so at any time the ellipse is

$$
\frac{x^{2}}{a^{2}}+\frac{y^{2}}{b^{2}}=1
$$

The area must remain constant since the flow is incompressible, and we take $a(t) b(t)=1$ to preserve the area. To be specific, we take

$$
a(t)=1+\frac{1}{4} \cos \omega t
$$

with some frequency $\omega$ so that $3 / 4 \leq a \leq 5 / 4$ and $4 / 5 \leq b \leq 4 / 3$, and thus the ellipse stays well within the central half-square $-\pi / 2 \leq x, y \leq \pi / 2$ of $Q$. We assume that the points move along rays $\theta=$ constant, so that the velocity of a point on the ellipse is

$$
\left(a^{\prime} \cos \theta, b^{\prime} \sin \theta\right)
$$

We note for later use that

$$
\sigma \equiv d s / d \theta=\sqrt{a^{2} \sin \theta^{2}+b^{2} \cos \theta^{2}}=\sqrt{b^{4} x^{2}+a^{4} y^{2}}
$$

where $s=$ arclength. Furthermore, the unit tangent and normal vectors to this curve are

$$
\tau=\left(-a^{2} y, b^{2} x\right) / \sigma, \quad n=\left(b^{2} x, a^{2} y\right) / \sigma,
$$

$\partial \tau / \partial s=-\kappa n$ and the curvature is given by $\kappa=\sigma^{-3}$.

The next step is to find a velocity field which gives the motion specified for the ellipse. We choose a stream function $\psi^{(0)}$ and then define the velocity as $\left(\psi_{y}^{(0)},-\psi_{x}^{(0)}\right)$, so that the divergence is zero. Note that $a^{\prime} / a=-b^{\prime} / b$ since $a b=1$. For $-\pi / 2 \leq x, y \leq \pi / 2$ we define

$$
\psi^{(0)}=\frac{a^{\prime}}{a} x y
$$

so that

$$
v^{(0)}=\left(\frac{a^{\prime}}{a} x, \frac{b^{\prime}}{b} y\right)
$$

in this smaller region, matching the velocity already specified on the curve. Later we extend $\psi^{(0)}$ to the whole box $Q$ so that it is $2 \pi$-periodic in each variable. We note for later use that $\Delta v^{(0)}=0$. 
We suppose that the equilibrium state of the membrane is the circle of radius $1 / 2$, with total length $\pi$, so that $\alpha=\theta / 2$ is a material coordinate. Then $d s / d \alpha=(d s / d \theta)(d \theta / d \alpha)=2 \sigma$. The force $f$ on the curve is given in terms of $\tau, n$, and $s_{\alpha}=d s / d \alpha$ by

$$
f=\partial_{s}\left[\left(s_{\alpha}-1\right) \tau\right]=2 \partial_{s}\left(s_{\theta}\right) \tau-\left(2 s_{\theta}-1\right) \kappa n=f_{\tau} \tau+f_{n} n
$$

with

$$
f_{\tau}=2 \partial_{s}\left(s_{\theta}\right), \quad f_{n}=-\left(2 s_{\theta}-1\right) \kappa
$$

and since $\sigma=s_{\theta}$,

$$
\begin{gathered}
f_{\tau}=2 s_{\theta \theta} / \sigma=2 \sigma^{-2}\left(a^{2}-b^{2}\right) x y \\
f_{n}=-\sigma^{-3}(2 \sigma-1)=-2 \sigma^{-2}+\sigma^{-3} .
\end{gathered}
$$

We must choose $v$ and $p$ with the jump conditions (outside minus inside)

$$
f=[p] n-\nu\left[\frac{\partial v}{\partial n}\right]
$$

or, separating tangent and normal components,

$$
\left[\frac{\partial v}{\partial n}\right]=-f_{\tau} \tau, \quad[p]=f_{n} .
$$

To create tangential force on the boundary, we will add a term to the velocity inside the ellipse, giving the requisite jump in normal velocity. Since the velocity must be continuous, the new term must be zero on the curve. We again define it through a stream function. We define $\rho$ by

$$
\rho^{2}=\frac{x^{2}}{a^{2}}+\frac{y^{2}}{b^{2}}
$$

so that the ellipse corresponds to $\rho=1$. Let $g$ be any function defined near the ellipse. Define

$$
\psi^{(1)}=g\left(\rho^{2}-1\right)^{2}, \quad v^{(1)}=\left(\psi_{y}^{(1)},-\psi_{x}^{(1)}\right) .
$$

For any choice of $g$, the velocity will be continuous with a jump in $\partial v / \partial n$ depending on $g$. We find $\Delta\left(\rho^{2}-1\right)^{2}=8 \sigma^{2}$. Since $\psi=0$ and $\nabla \psi=0$ on the ellipse we get

$$
\frac{\partial v^{(1)}}{\partial n}=-g\left(\Delta\left(\rho^{2}-1\right)^{2}\right) \tau=-8 \sigma^{2} g \tau \text {. }
$$

Looking at $f_{\tau}$ above, we need $-8 \sigma^{2} g=f_{\tau}$ on the ellipse, or

$$
g=-\frac{1}{4 \sigma^{4}}\left(a^{2}-b^{2}\right) \sin \theta \cos \theta=-\sigma^{-4}\left(a^{2}-b^{2}\right) \frac{x y}{4 a b}
$$


on the ellipse. We will extend $\sigma$ positively to the interior $\rho<1$ and then define, for $\rho<1$,

$$
\psi^{(1)}=-\sigma^{-4}\left(a^{2}-b^{2}\right) \frac{x y}{4 a b}\left(\rho^{2}-1\right)^{2}
$$

Similarly, we define $p$ inside as the extension of $-f_{n}=\sigma^{-3}(2 \sigma-1)$, with $p=0$ outside, so that the jump condition for pressure in Eq. (6.14) is satisfied. We have now defined the velocity and pressure so that the ellipse moves with the fluid velocity and the jump conditions (6.14) hold.

To extend $\sigma$ to $\rho<1$, keeping it positive, we define $\sigma^{2}$ inside as

$$
\sigma^{2}=1+\left(a^{2}-1\right) \frac{y^{2}}{b^{2}}+\left(b^{2}-1\right) \frac{x^{2}}{a^{2}}
$$

Then $\sigma^{2} \rightarrow 1$ as $(x, y) \rightarrow(0,0)$. Also

$$
\sigma^{2}=1-\rho^{2}+a^{2} \frac{y^{2}}{b^{2}}+b^{2} \frac{x^{2}}{a^{2}}
$$

and for $\rho=1$ this agrees with Eq. (6.5) since $a b=1$. We check $\sigma$ is strictly positive: Choose $c_{0}>0$ so that $a, b \geq c_{0}$ at each time. (For our example, $c_{0}=3 / 4$.) Then

$$
\sigma^{2} \geq 1-\rho^{2}+c_{0} \rho^{2}=1-\left(1-c_{0}\right) \rho^{2} \geq c_{0}
$$

for $\rho^{2} \leq 1$, as desired.

We need third derivatives of $\psi^{(1)}$, since $v^{(1)}=\left(\psi_{y}^{(1)},-\psi_{x}^{(1)}\right)$ and the body force depends on $\Delta v^{(1)}$. To record various derivatives, we write

$$
\begin{array}{r}
\psi^{(1)}=C_{0} x y Q R, \quad C_{0}=\left(b^{2}-a^{2}\right) /(4 a b), \\
Q=\sigma^{-4}, \quad R=\left(\rho^{2}-1\right)^{2}
\end{array}
$$

with $\sigma$ extended to $\rho \leq 1$ as in Eqs. (6.20) and (6.21). For convenience write

$$
\rho^{2}=b^{2} x^{2}+a^{2} y^{2}, \quad \sigma^{2}=1+b^{2}\left(b^{2}-1\right) x^{2}+a^{2}\left(a^{2}-1\right) y^{2}
$$

or

$$
\sigma^{2}=1+B x^{2}+A y^{2}, \quad B=b^{2}\left(b^{2}-1\right), \quad A=a^{2}\left(a^{2}-1\right)
$$

Then

$$
\begin{aligned}
& R_{x}=4\left(\rho^{2}-1\right) b^{2} x, \quad R_{y}=4\left(\rho^{2}-1\right) a^{2} y, \quad R_{x y}=8 x y \\
& R_{x x}=8 b^{4} x^{2}+4\left(\rho^{2}-1\right) b^{2}, \quad R_{y y}=8 a^{4} y^{2}+4\left(\rho^{2}-1\right) a^{2} \\
& \Delta R_{x}=8 x\left(1+3 b^{4}\right), \quad \Delta R_{y}=8 y\left(1+3 a^{4}\right)
\end{aligned}
$$




$$
\begin{aligned}
& Q_{x}=-4 \sigma^{-6} B x, \quad Q_{y}=-4 \sigma^{-6} A y, \quad Q_{x y}=24 \sigma^{-8} A B x y \\
& Q_{x x}=4 \sigma^{-8} B\left(5 B x^{2}-1-A y^{2}\right), \quad Q_{y y}=4 \sigma^{-8} A\left(5 A y^{2}-1-B x^{2}\right) \\
& \Delta Q_{x}=24 \sigma^{-10} B x\left(3 B-5 B^{2} x^{2}+3 A B y^{2}+A+A B x^{2}-7 A^{2} y^{2}\right) \\
& \Delta Q_{y}=24 \sigma^{-10} A y\left(3 A-5 A^{2} y^{2}+3 A B x^{2}+B+A B y^{2}-7 B^{2} x^{2}\right)
\end{aligned}
$$

Using derivatives of $R$ and $Q$ we can write

$$
\begin{aligned}
& v_{1}^{(1)}=\psi_{y}^{(1)}=C_{0} x Q R+C_{0} x y\left(Q_{y} R+Q R_{y}\right) \\
& v_{2}^{(1)}=-\psi_{x}^{(1)}=-C_{0} y Q R-C_{0} x y\left(Q_{x} R+Q R_{x}\right)
\end{aligned}
$$

$$
\begin{aligned}
\Delta v_{1}^{(1)} / C_{0} & =\Delta \psi_{y}^{(1)} / C_{0}=2 Q R_{x}+2 Q_{x} R \\
& +2 y\left(Q_{x y} R+Q_{x} R_{y}+Q R_{x y}+Q_{y} R_{x}\right) \\
& +x\left(6 Q_{y} R_{y}+3 Q R_{y y}+3 Q_{y y} R+Q_{x x} R+Q R_{x x}+2 Q_{x} R_{x}\right) \\
& +x y\left(\left(\Delta Q_{y}\right) R+3 Q_{y y} R_{y}+3 Q_{y} R_{y y}+Q \Delta R_{y}\right. \\
& \left.+Q_{x x} R_{y}+2 Q_{x y} R_{x}+2 Q_{x} R_{x y}+Q_{y} R_{x x}\right) \\
-\Delta v_{2}^{(1)} / C_{0} & =\Delta \psi_{x}^{(1)} / C_{0}=2 Q R_{y}+2 Q_{y} R \\
& +2 x\left(Q_{x y} R+Q_{y} R_{x}+Q R_{x y}+Q_{x} R_{y}\right) \\
& +y\left(6 Q_{x} R_{x}+3 Q R_{x x}+3 Q_{x x} R+Q_{y y} R+Q R_{y y}+2 Q_{y} R_{y}\right) \\
& +x y\left(\left(\Delta Q_{x}\right) R+3 Q_{x x} R_{x}+3 Q_{x} R_{x x}+Q \Delta R_{x}\right. \\
& \left.+Q_{y y} R_{x}+2 Q_{x y} R_{y}+2 Q_{y} R_{x y}+Q_{x} R_{y y}\right)
\end{aligned}
$$

With these pieces in place, we can write explicitly the velocity $v$, pressure $p$, and body forces, inside and outside the ellipse. Inside we use Eqs. (6.8), (6.34) and (6.35) to define

$$
v=v^{(0)}+v^{(1)}, \quad \rho<1
$$

and $p$ as stated before, using the extended $\sigma$ in Eq. (6.20),

$$
p=\sigma^{-3}(2 \sigma-1), \quad \rho<1
$$

so that

$$
\nabla p=\sigma^{-5}(3-4 \sigma)(B x, A y), \quad \rho<1 .
$$

To define $v$ outside the ellipse, we use a periodic extension of $\zeta(x)=x$ for $|x| \leq \pi / 2$, extended to $|x| \leq \pi$, as defined below. Outside the ellipse we define, in place of (6.7),

$$
\psi^{(0)}(x, y)=\frac{a^{\prime}}{a} \zeta(x) \zeta(y)
$$


so that the outside velocity is

$$
v=\left(\psi_{y}^{(0)},-\psi_{x}^{(0)}\right)=\frac{a^{\prime}}{a}\left(\zeta(x) \zeta^{\prime}(y),-\zeta^{\prime}(x) \zeta(y)\right), \quad \rho>1
$$

We take the pressure to be zero outside,

$$
p=0, \quad \rho>1 .
$$

To use this example as a test problem, we need to give the nonhomogeneous terms, or body force, as well as the force on the boundary, in order to compute $v$ and $p$, and compare with the exact ones above. The Stokes equations (1.1) with force (1.2) $F=f \delta_{\Gamma}+F_{b}$ where $F_{b}$ is the body force (interior and exterior) and $f \delta_{\Gamma}$ is the force on the boundary. The boundary force is $f=f_{n} n+f_{\tau} \tau$, with $n, \tau$ given by Eq. (6.8) and $f_{n}, f_{\tau}$ given by Eq. (6.10) or Eqs. (6.11) and (6.12). As for the body force $F_{b}$, we have with the choices above,

$$
F_{b}=-\nu \Delta v^{(1)}+\nabla p, \quad \rho<1
$$

and

$$
F_{b}=-\nu\left(\Delta \psi_{y}^{(0)},-\Delta \psi_{x}^{(0)}\right), \quad \rho>1 .
$$

Inside, the components of $F_{b}$ are given by Eqs. (6.36), (6.37) and (6.40), taking into account the factors $C_{0}$ and $-\nu$.) Outside they are

$$
\begin{aligned}
& F_{b 1}=-\nu \frac{a^{\prime}}{a}\left(\zeta^{\prime \prime}(x) \zeta^{\prime}(y)+\zeta(x) \zeta^{\prime \prime \prime}(y)\right), \quad \rho>1 \\
& F_{b 2}=\nu \frac{a^{\prime}}{a}\left(\zeta^{\prime \prime \prime}(x) \zeta(y)+\zeta^{\prime}(x) \zeta^{\prime \prime}(y)\right), \quad \rho>1
\end{aligned}
$$

Finally we give the definition of $\zeta$. We first construct an odd polynomial $q(x)$ on $[-\pi / 2, \pi / 2]$, so that after translation $\zeta$ will be odd about $x= \pm \pi$. We specify $q^{\prime}(\pi / 2)=1 ; q^{\prime \prime}(\pi / 2)=0 ; q^{\prime \prime \prime}(\pi / 2)=0$. With

$$
q(x)=c_{1} x+c_{2} x^{3}+c_{3} x^{5}+c_{4} x^{7}
$$

we get

$$
c_{1}=-\frac{27}{8}, \quad c_{2}=\frac{35}{2 \pi^{2}}, \quad c_{3}=-\frac{42}{\pi^{4}}, \quad c_{4}=\frac{40}{\pi^{6}}
$$

Now define

$$
\begin{gathered}
\zeta(x)=x, \quad-\pi / 2 \leq x \leq \pi / 2 \\
\zeta(x)=q(x-\pi), \quad \pi / 2 \leq x \leq \pi \\
\zeta(x)=q(x+\pi), \quad-\pi \leq x \leq-\pi / 2
\end{gathered}
$$

We have defined $\zeta$ so that $\zeta^{\prime \prime \prime}$ is continuous but not differentiable at $x= \pm \pi / 2$. Thus the body force $F_{b}$ has the same property. 


\section{Numerical results}

The algorithm of Sec. 3 was implemented in the C programming language and tested on a collection of interfacial Stokes flows, including the exact elastic elliptical solution derived in Sec. 6 and shown in Fig. 3. We present quantitative evidence of its robustness and second-order accuracy. Our current version is suboptimally efficient since the $p^{2}$ Fourier coefficients of the body force are computed in $O\left(p^{2} N_{\Gamma}\right)$ time when the interface contains $N_{\Gamma}$ elements, so we do not report CPU times. Once geometric nonuniform FFT techniques [35]

are incorporated, the computational cost for the velocity on the interface and the interface motion at one time step will be about $O\left(N_{\Gamma}\right)$. To compute the velocity field on a grid at one time will add a cost proportional to the number of grid points. The overall complexity of our algorithm will then be optimal, and large-scale simulations will be practical.

Table 1 presents 1-norm errors

$$
E_{\Gamma}=\frac{1}{N_{\Gamma}} \sum_{j=1}^{N_{\Gamma}}\left|\varphi\left(\gamma_{j}, t\right)\right|
$$

and

$$
E_{v}=\frac{1}{N^{2}} \sum_{i=1}^{N} \sum_{j=1}^{N}\left\|v_{i j}-\tilde{v}_{i j}\right\|
$$

in the interface location and fluid velocity respectively, after $S$ time steps up to the final time $T=11$ corresponding to one full cycle of the elliptical motion. Here $\Gamma$ is the computed interface, composed of line segments with $N_{\Gamma}$ endpoints $\gamma_{j}, \varphi$ is the signed distance to the exact interface at time $T$, and $v_{i j}$ and $\tilde{v}_{i j}$ are the exact and computed fluid velocities at points $(i, j)$ of a uniform $N \times N$ grid. An $L$-level distance tree was employed as a basis for contouring, with $D$ steps of subgrid resolution to tolerance $\epsilon=2^{-D}$. The velocity evaluation used $p \times p$ Fourier modes, while three different smoothing parameters $\sigma, \sigma / 2$ and $\sigma / 8$ were tested, and shown in successive columns in Table 1, to ensure convergence of the local correction. Since the velocity is of order unity, the absolute error is reported. Errors were also measured in the maximum norm; for the velocity, the maximum and 1-norm errors were about the same, while the maximum interface errors were about a factor of two or three larger. The interface errors measured at segment midpoints were also within ten percent of the reported errors. The area $\pi$ of the ellipse is conserved by the exact flow. In these tests the area did not vary much over time; for the bottom row in Table 1 the error in the computed area was about .003 , or $.1 \%$, regardless of $\sigma$.

Table 1 supports several conclusions about the convergence rate of our methods as a function of the time step $k=T / S$, the average interface resolution 
Step 0

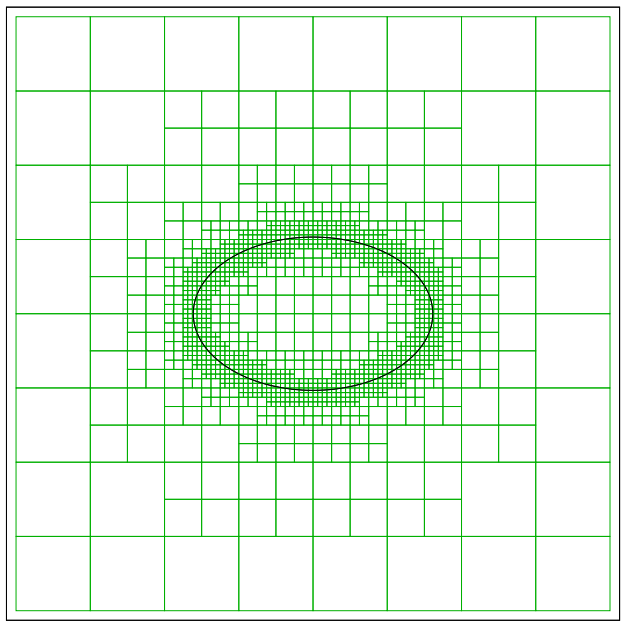

44

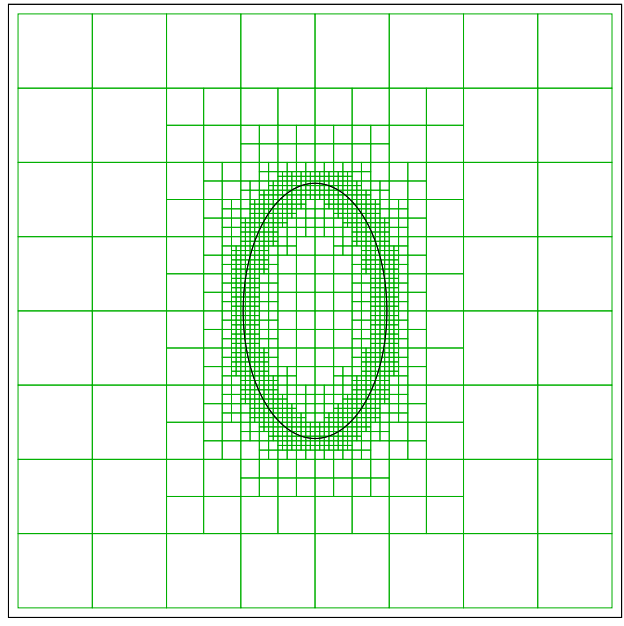

22

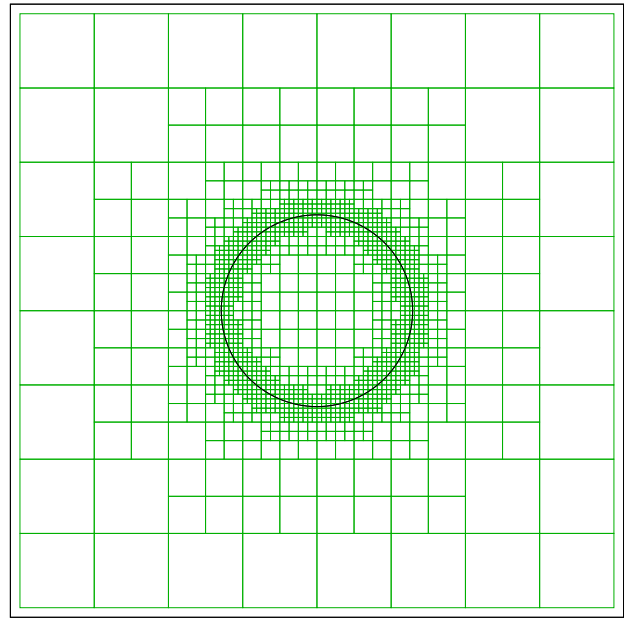

88

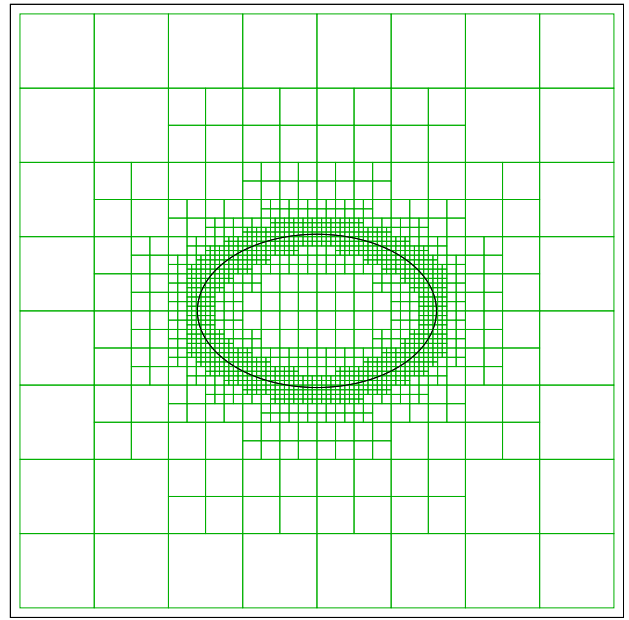

Fig. 3. Moving elastic elliptical interfaces and 7-level quadtrees which organize them for efficient distance evaluation.

$h=|\Gamma| / N_{\Gamma} \approx 2^{-L-D}$, and the velocity resolution control parameters $p$ and $\sigma$. We can verify second-order convergence in $k$ by selecting entries in which the $O\left(p^{2}\right)$ and $O(\sigma)$ quadrature and Ewald summation errors decrease faster than $O\left(k^{2}+h^{2}\right)$. For example, as we proceed down the first column with the largest $\sigma$, the interface location error decreases by factors of approximately four from .02200 through .00495 and .00098 to .00036 , while the velocity error decreases similarly from .07180 through .02260 and .00261 to .00097 , as $S$ doubles from 22 to $176, p$ doubles from 15 to 80 , and $L+D$ increases from 8 to 14 . Factors near four can be seen more clearly by proceeeding diagonally, e.g., with $S=44, p=20, \sigma=.064 ; S=88, p=30, \sigma=.016 ; S=176$, $p=80, \sigma=.0005$. Thus second-order convergence in time is verified. The asymptotic order of convergence in the Ewald parameters $p$ and $\sigma$ is more complicated, because it is often dominated by the other discretization errors. 
Table 1

Interface and velocity errors $E_{\Gamma}$ and $E_{v}$ after one oscillation of an elastic ellipse.

\begin{tabular}{|c|c|c|c|c|c|c|c|c|c|c|c|c|}
\hline$L$ & $D$ & $S$ & $p$ & $\sigma$ & $E_{\Gamma}$ & $E_{v}$ & $\sigma$ & $E_{\Gamma}$ & $E_{v}$ & $\sigma$ & $E_{\Gamma}$ & $E_{v}$ \\
\hline \multirow[t]{3}{*}{6} & 2 & 22 & 15 & .128 & .02200 & .07180 & .064 & .01610 & .05240 & .0160 & .01040 & .02130 \\
\hline & & & 20 & .064 & .00675 & .02380 & .032 & .00420 & .01280 & .0080 & .00484 & .01440 \\
\hline & & & 30 & .032 & .00394 & .01210 & .016 & .00390 & .01050 & .0040 & .00387 & .01380 \\
\hline \multirow[t]{3}{*}{7} & 3 & 44 & 20 & .064 & .00495 & .02260 & .032 & .00299 & .01140 & .0080 & .00237 & .01120 \\
\hline & & & 30 & .032 & .00213 & .01020 & .016 & .00154 & .00640 & .0040 & .00174 & .00932 \\
\hline & & & 40 & .016 & .00154 & .00327 & .008 & .00155 & .00455 & .0020 & .00160 & .00646 \\
\hline \multirow[t]{3}{*}{8} & 4 & 88 & 30 & .032 & .00196 & .00956 & .016 & .00125 & .00557 & .0040 & .00116 & .00838 \\
\hline & & & 40 & .016 & .00098 & .00261 & .008 & .00082 & .00310 & .0020 & .00079 & .00502 \\
\hline & & & 60 & .008 & .00071 & .00158 & .004 & .00072 & .00257 & .0010 & .00039 & .00288 \\
\hline \multirow[t]{3}{*}{9} & 5 & 176 & 40 & .016 & .00089 & .00245 & .008 & .00054 & .00265 & .0020 & .00047 & .00430 \\
\hline & & & 60 & .008 & .00037 & .00085 & .004 & .00036 & .00127 & .0010 & .00035 & .00180 \\
\hline & & & 80 & .004 & .00036 & .00097 & .002 & .00036 & .00139 & .0005 & .00037 & .00160 \\
\hline
\end{tabular}

Thus we report velocity errors $E_{v}$ at the initial time $t=0$, where the time discretization error is irrelevant, in Table 2. The $O\left(\delta^{3}\right)$ convergence predicted by Eq. (5.50) is evident in the approximate factor of eight decrease in the first column of errors from 0.0417 through .0184 and .00259 to .000328 as $\sigma=\delta^{2} / 4$ is reduced by factors of four from .128 to .004 .

We also verified topological robustness and numerical accuracy on a variety of more complicated flows, with samples shown in Figures 4 and 5. Detailed convergence studies by parameter refinement also support the conclusions above. In addition, Figure 4 demonstrates that the connectivity of the initial interface is often preserved under sufficiently accurate computations of Stokes flow. The initial interface has three holes, which are preserved as they become circular in the Stokes flow.

Figure 5 demonstrates the effect of a body force

$$
F_{b}\left(x_{1}, x_{2}\right)=\frac{1}{4}\left(-\sin 2 x_{2}, \cos 2 x_{1}\right)
$$

simulating a shearing flow around the box center. Interfaces in such flows are subject to competing influences, since the Stokes flow driven by curvature of the interface tends to circularize the interfaces, while the shearing body force tends to spin them into complex shapes. In this example, the initial complex interfaces rapidly become circular under Stokes flow, but then collide and merge into a larger circular bubble. Thus connectivity is not always conserved 
Table 2

Velocity errors $E_{v}$ at initial time $t=0$.

\begin{tabular}{|c|c|c|c|c|c|c|c|c|c|c|}
\hline$L$ & $D$ & $p$ & $\sigma$ & $E_{v}$ & $\sigma$ & $E_{v}$ & $\sigma$ & $E_{v}$ & $\sigma$ & $E_{v}$ \\
\hline \multirow[t]{3}{*}{6} & 2 & 15 & .128 & .0417 & .064 & .0227 & .032 & .0141 & .0160 & .013 \\
\hline & & 20 & .064 & .0178 & .032 & .00997 & .016 & .0074 & .0080 & .00926 \\
\hline & & 30 & .032 & .00848 & .016 & .0048 & .008 & .0057 & .0040 & .00654 \\
\hline \multirow[t]{3}{*}{7} & 3 & 20 & .064 & .0184 & .032 & .0101 & .016 & .00738 & .0080 & .00919 \\
\hline & & 30 & .032 & .00864 & .016 & .00485 & .008 & .00573 & .0040 & .00661 \\
\hline & & 40 & .016 & .00233 & .008 & .00237 & .004 & .00344 & .0020 & .00381 \\
\hline \multirow[t]{3}{*}{8} & 4 & 30 & .032 & .00923 & .016 & .00525 & .008 & .00605 & .0040 & .00698 \\
\hline & & 40 & .016 & .00259 & .008 & .0025 & .004 & .00358 & .0020 & .00396 \\
\hline & & 60 & .008 & .000884 & .004 & .000682 & .002 & .00107 & .0010 & .00115 \\
\hline \multirow[t]{3}{*}{9} & 5 & 40 & .016 & .0026 & .008 & .00254 & .004 & .00362 & .0020 & .004 \\
\hline & & 60 & .008 & .000883 & .004 & .000688 & .002 & .00108 & .0010 & .00116 \\
\hline & & 80 & .004 & .000328 & .002 & .000675 & .001 & .000862 & .0005 & .00085 \\
\hline
\end{tabular}

in this model. It appears that the higher-order derivatives involved in the interface forces $f_{\Gamma}$ take effect over shorter time scales than the body forces $F_{b}$, and therefore the smoothing effect of the singular Stokes flow dominates the interfacial evolution when computed with sufficient accuracy. Here, Table 3 shows that area is eventually conserved to about $1 \%$ accuracy, a reasonable result for the change in connectivity of this singular interface.

Table 3

Area $A$ enclosed by two merging propellers at the initial and final times.

\begin{tabular}{ccccc|cc|cc|cc}
\hline$S$ & $L$ & $D$ & $p$ & $A_{0}$ & $\sigma$ & $A$ & $\sigma$ & $A$ & $\sigma$ & $A$ \\
\hline 88 & 6 & 2 & 30 & 1.299362 & .0160 & 1.08076 & .0080 & 1.15143 & .0040 & 1.14558 \\
176 & 7 & 3 & 40 & 1.331076 & .0080 & 1.22488 & .0040 & 1.24753 & .0020 & 1.24803 \\
352 & 8 & 4 & 60 & 1.326567 & .0040 & 1.28719 & .0020 & 1.29007 & .0010 & 1.29088 \\
704 & 9 & 5 & 80 & 1.327284 & .0020 & 1.30807 & .0010 & 1.30923 & .0005 & 1.31007 \\
\hline
\end{tabular}

\section{Future Directions}

The algorithm used in this work extends directly to three dimensions. If the force on a moving surface is determined by stretching of area, the description of the evolution in Sec. 2 applies directly. The Stokes velocity resulting from the interfacial force is a single layer potential on the surface with $1 / r$ singularity. 
Step 0

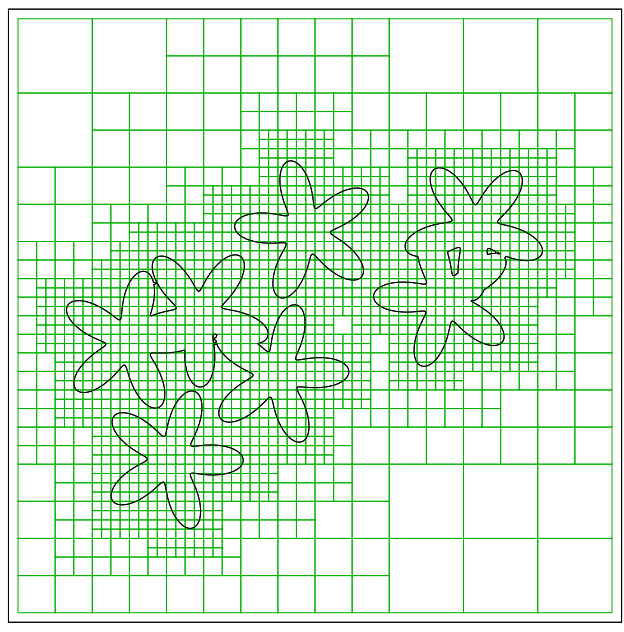

30

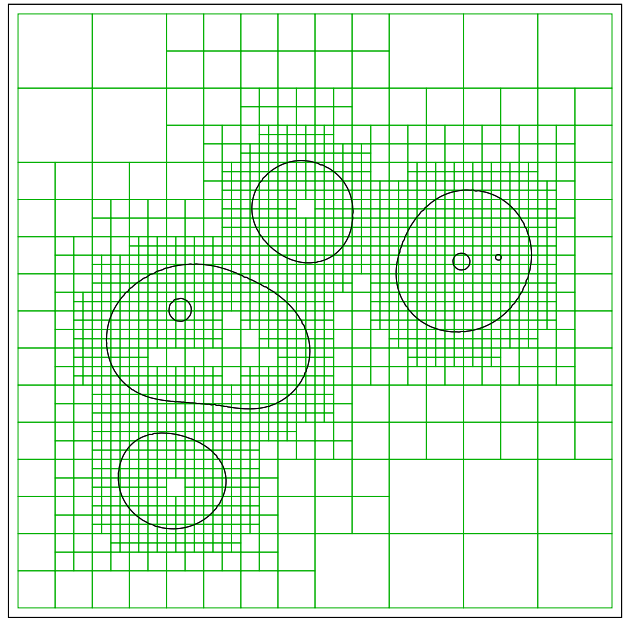

10

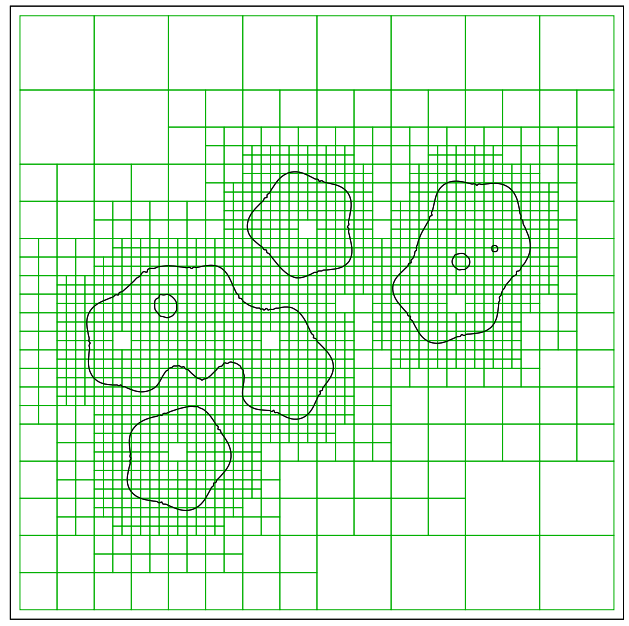

300

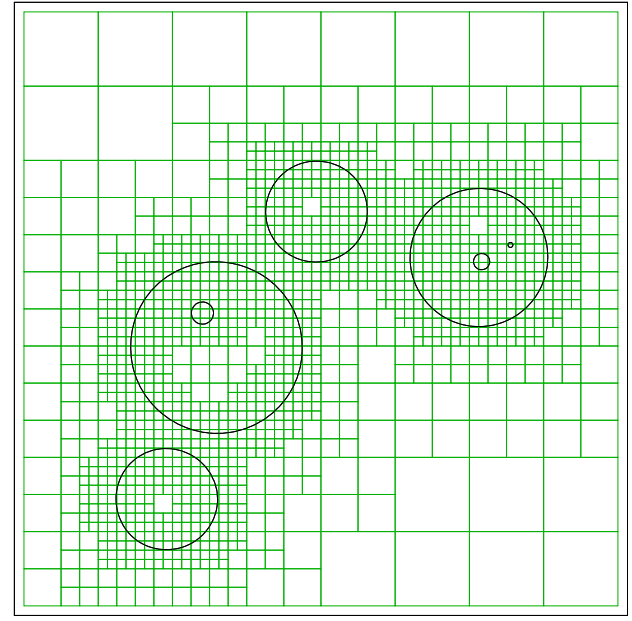

Fig. 4. Complex elastic interfaces evolving under Stokes flow.

The Ewald splitting applies to the fundamental solution, and the treatment of the Fourier part of the integral is the same [36]. The local part can be approximated as in Sec. 5; a method of this type for surface layer potentials for the Laplacian was developed in [3] and a general approach constructed in [42]. Semi-Lagrangian contouring in three dimensions has been implemented in an open-source code [2].

We have assumed that the viscosity is the same on both sides of the interface. Boundary integral methods have been used for Stokes flow with different viscosities (e.g. see $[31,32]$ ). The calculation of the velocity determined by the force on the moving boundary requires the solution of an integral equation on the boundary as a preliminary step (e.g., see [31,32,5]). The present method should extend to the case of differing viscosities in this way.

The Stokes equations (1.1) describe fluid flow only for very low Reynolds 
Step 0

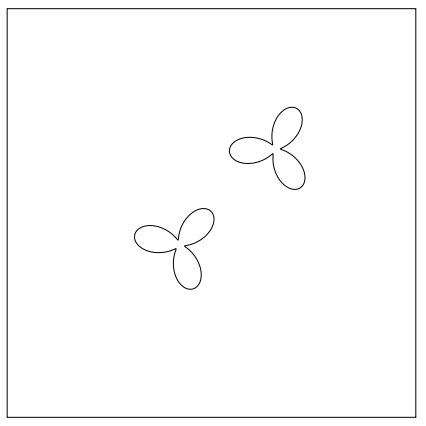

250

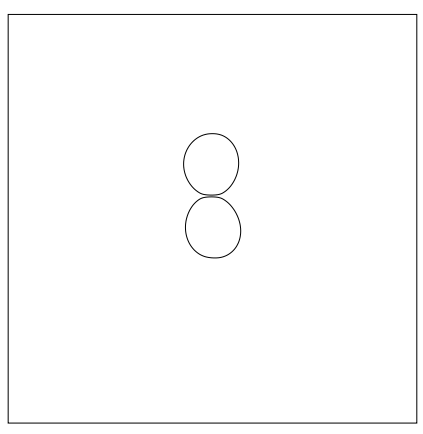

280

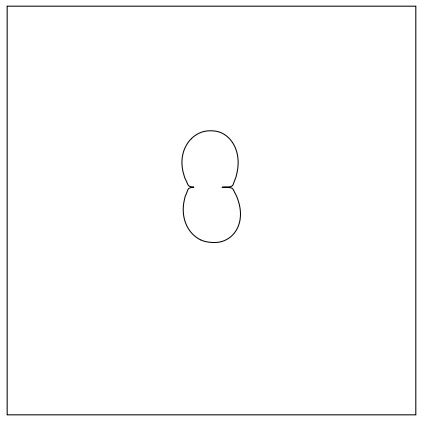

50

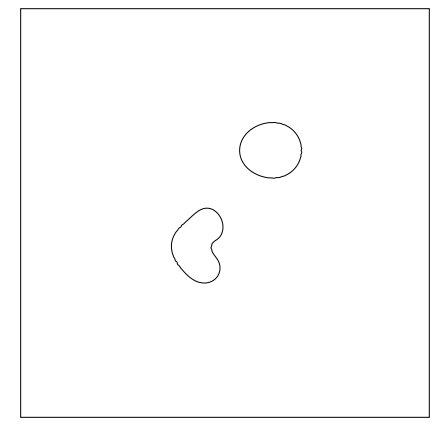

260

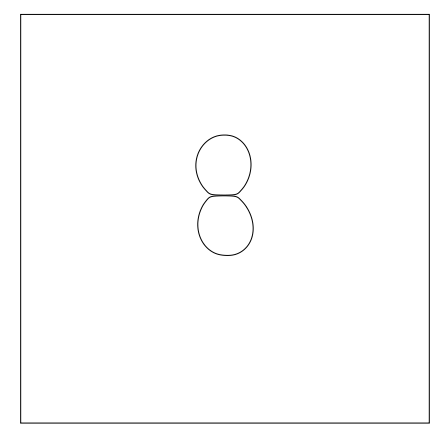

330

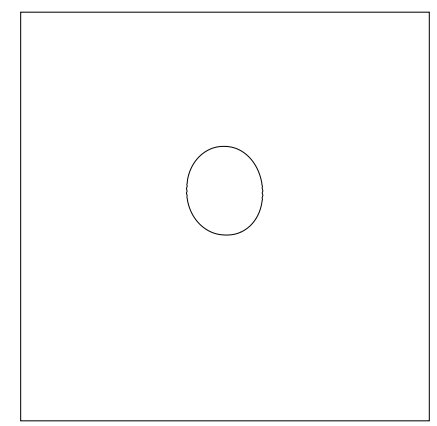

150

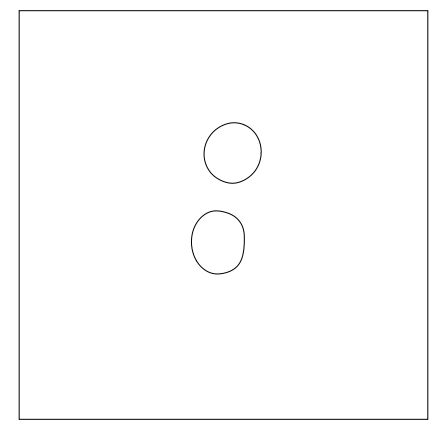

270

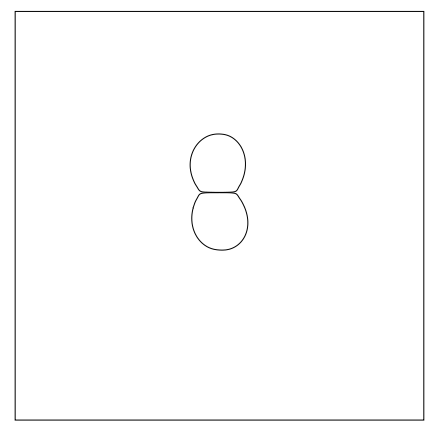

630

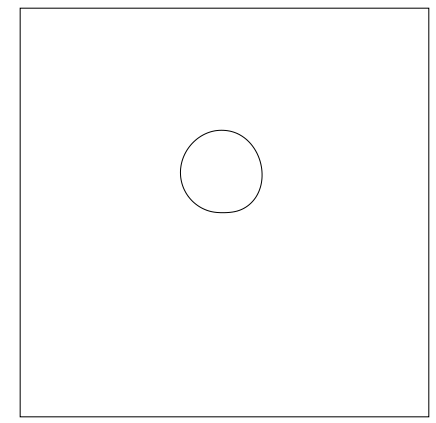

Fig. 5. Interfacial Stokes flow with a simulated shearing force.

number. In modeling flows with higher Reynolds numbers, Eqs. (1.1) should be replaced by the Navier-Stokes equations

$$
v_{t}+v \cdot \nabla v+\nabla p=\nu \Delta v+f \delta_{\Gamma}, \quad \nabla \cdot v=0 .
$$

We expect the present method can be extended to Navier-Stokes flow with an elastic interface. One way to proceed begins with the observation that the jump conditions (1.6) are the same in the two cases. At each time we can write the Navier-Stokes velocity $v$ determined by Eqs. (8.1) as a sum $v=v^{s}+v^{r}$ where $v^{s}$ is the Stokes velocity determined by Eq. (1.1). Then $v^{r}$ is $C^{1}$ at the interface; the jump in the normal derivative has been removed. The evolution equation for the regular part $v^{r}$ is similar to Eq. (8.1), without the interfacial force, but with a forcing term determined by $v^{s}$. Since the singular interfacial 
force has been removed to the Stokes term $v^{s}$, it should be possible to solve for the smoother velocity $v^{r}$ accurately on a regular grid. With this additional step, the method presented here for Stokes flow could be used to solve the Navier-Stokes equations (8.1).

\section{Acknowledgements}

This material is based upon work supported by the National Science Foundation under grants DMS-0404765 (Beale) and DMS-0512963 (Strain), and by the Air Force Office of Scientific Research, Air Force Materiel Command, USAF, under grant number FA9550-05-1-0120 (Strain). The U.S. Government is authorized to reproduce and distribute reprints for Governmental purposes notwithstanding any copyright notation thereon.

\section{References}

[1] D. J. Adams, G. S. Dubey, Taming the Ewald sum in the computer simulation of charged systems, J. Comput. Phys. 72 (1987) 156-176.

[2] A. Bargteil, T. G. Goktekin, J. F. O'Brien, J. Strain, A semi-Lagrangian contouring method for fluid simulation, ACM Trans. Graphics 25 (2006) 19-38.

[3] J. T. Beale, A grid-based boundary integral method for elliptic problems in three dimensions, SIAM J. Numer. Anal. 42 (2004) 599-620.

[4] J. T. Beale, M.-C. Lai, A method for computing nearly singular integrals, SIAM J. Numer. Anal. 38 (2001) 1902-25.

[5] J. T. Beale, A. Layton, On the accuracy of finite difference methods for elliptic problems with interfaces, Commun. Appl. Math. Comput. Sci. 1 (2006) 91-119.

[6] G. Biros, L. Ying, D. Zorin, An embedded boundary integral solver for the unsteady incompressible Navier-Stokes equations, J. Comput. Phys. (2004) 121141.

[7] G. Biros, L. Ying, D. Zorin, A fast solver for the Stokes equations with distributed forces in complex geometries, J. Comput. Phys. 193 (2004) 317348.

[8] R. Cortez, The method of regularized Stokeslets, SIAM J. Sci. Comput. 23 (2001) 1204-1225.

[9] R. Cortez, M. Minion, The blob projection method for immersed boundary problems, J. Comput. Phys. 161 (2000) 428-453. 
[10] G.-H. Cottet, E. Maitre, A level-set formulation of immersed boundary methods for fluid-structure interaction problems, C. R. Math. Acad. Sci. Paris 338 (2004) $581-586$.

[11] G.-H. Cottet, E. Maitre, A level-set method for fluid-structure interactions with immersed surfaces, Mathematical Models and Methods in the Applied Sciences 16 (2006) 415-438.

[12] H. Dym, H. P. McKean, Fourier Series and Integrals, Academic Press, Toronto, 1972.

[13] P. Ewald, Die Berechnung optischer und elektrostatistischer Gitterpotentiale, Ann. Phys. 64 (1921) 253.

[14] G. B. Folland, Introduction to Partial Differential Equations, 2nd ed., Princeton University Press, 1995.

[15] L. Greengard, M. Kropinski, Integral equation methods for Stokes flow in doubly-periodic domains, J. Engrg. Math. 48 (2004) 157-70.

[16] L. Greengard, J. Strain, A fast algorithm for heat potentials, Commun. Pure Appl Math. 43 (1990) 949-63.

[17] B. Griffith, R. Hornung, D. McQueen, C. Peskin, An adaptive, formally second order accurate version of the immersed boundary method, J. Comput. Phys. 223 (2007) 10-49.

[18] B. Griffith, C. Peskin, On the order of accuracy of the immersed bondary method: Higher order convergence rates for sufficiently smooth problems, J. Comput. Phys. 208 (2005) 75-105.

[19] H. Hasimoto, On the periodic fundamental solutions of the Stokes equations and their applications to viscous flow past a cubic array of spheres, J. Fluid Mech. 5 (1959) 317-326.

[20] T. Y. Hou, Z. Shi, Removing the stiffness of elastic force from the immersed boundary method for the 2d Stokes equations, preprint.

[21] D. D. Joseph, Y. Y. Renardy, Fundamentals of Two-Fluid Dynamics, Part I: Mathematical Theory and Applications, Springer, New York, 1993.

[22] M. Kropinski, An efficient numerical method for studying interfacial motion in two-dimensional creeping flows, J. Comput. Phys. 171 (2001) 479-508.

[23] D. Le, B. Khoo, J. Peraire, An immersed interface method for viscous incompressible flows involving rigid and flexible boundaries, J. Comput. Phys. 220 (2006) 109-38.

[24] L. G. Leal, Laminar Flow and Convective Transport Processes, ButterworthHeinemann, Boston, 1992.

[25] L. Lee, R. LeVeque, An immersed interface method for incompressible NavierStokes equations, SIAM J. Sci. Comput. 25 (2003) 832-856. 
[26] R. J. LeVeque, Z. Li, Immersed interface methods for Stokes flow with elastic boundaries or surface tension, SIAM J. Sci. Comput. 18 (1997) 709-735.

[27] Z. Li, M.-C. Lai, The immersed interface method for the Navier-Stokes equations with singular forces, J. Comput. Phys. 171 (2001) 822-42.

[28] A. Mayo, C. Peskin, An implicit numerical method for fluid dynamics problems with immersed elastic boundaries, Contemp. Math. 141 (1993) 261-77.

[29] C. Peskin, The immersed boundary method, Acta Numer. 11 (2002) 479-517.

[30] C. Peskin, B. Printz, Improved volume conservation in the computation of flows with immersed elastic boundaries, J. Comput. Phys. 105 (1993) 33-46.

[31] C. Pozrikidis, Boundary Integral and Singularity Methods for Linearized Viscous Flow, Cambridge Univ. Press, Cambridge, 1992.

[32] C. Pozrikidis, Interfacial dynamics for Stokes flow, J. Comput. Phys. 169 (2001) 250-301.

[33] A. Roma, C. Peskin, M. Berger, An adaptive version of the immersed boundary method, J. Comput. Phys. 153 (1999) 509-34.

[34] D. Saintillan, E. S. G. Shaqfeh, E. Darve, A smooth particle-mesh Ewald algorithm for stokes suspension simulations: the sedimentation of fibres, Phys. Fluids 17 (2005) 033301-1-21.

[35] I. S. Sammis, J. Strain, GNUFFT: a geometric nonuniform fast Fourier transform, preprint (2007).

[36] J. Strain, Fast potential theory II: Layer potentials and discrete sums, J. Comput. Phys. 99 (1992) 251-270.

[37] J. Strain, Fast tree-based redistancing for level set computations, J. Comput. Phys. 152 (1999) 664-686.

[38] J. Strain, Semi-Lagrangian methods for level set equations, J. Comput. Phys. 151 (1999) 498-533.

[39] J. Strain, Tree methods for moving interfaces, J. Comput. Phys. 151 (1999) 616-648.

[40] J. Strain, A fast modular semi-Lagrangian method for moving interfaces, J. Comput. Phys. 161 (2000) 512-536.

[41] J. Strain, A fast semi-Lagrangian contouring method for moving interfaces, J. Comput. Phys. 170 (2001) 373-394.

[42] J. Strain, Locally-corrected spectral methods and overdetermined elliptic systems, J. Comput. Phys. 224 (2007) 1243-54.

[43] M. Sussman, K. Smith, M. Hussaini, M. Ohta, R. Zhi-Wei, A sharp interface method for incompressible two-phase flows, J. Comput. Phys. 221 (2007) 469505. 
[44] A.-K. Tornberg, M. Shelley, Simulating the dynamics and interactions of flexible fibers in Stokes flows, J. Comput. Phys. 196 (2005) 8-40.

[45] C. Tu, C. Peskin, Stability and instability in the computation of flows with moving immersed boundaries: a comparison of three methods, SIAM J. Sci. Statist. Comput. 13 (1992) 1361-1376.

[46] S. Unverdi, G. Tryggvason, A front-tracking method for viscous, incompressible, multi-fluid flows, J. Comput. Phys. 100 (1992) 25-37.

[47] S. Xu, Z. J. Wang, An immersed interface method for simulating the interaction of a fluid with moving boundaries, J. Comput. Phys. 216 (2006) 454-93.

[48] H.-K. Zhao, T. Chan, B. Merriman, S. Osher, A variational level set approach to multiphase motion, J. Comput. Phys. 127 (1996) 179-95. 